\title{
$q$-TENSOR SPACE AND $q$-WEYL MODULES
}

\author{
RICHARD DIPPER AND GORDON JAMES
}

\begin{abstract}
We obtain the irreducible representations of the $q$-Schur algebra, motivated by the fact that these representations give all the irreducible representations of $G L_{n}(q)$ in the nondescribing characteristic. The irreducible polynomial representations of the general linear groups in the describing characteristic are a special case of this construction.
\end{abstract}

The theory of polynomial representations of general linear groups is equivalent to the representation theory of Schur algebras (see Green's book [5] and the bibliography therein). In [4], we defined $q$-analogues of Schur algebras. When $q=1$, these are the usual Schur algebras, and when $q$ is a prime power, representations of $q$-Schur algebras give a substantial part of the representation theory of finite general linear groups in the nondescribing characteristic case, including all irreducible representations of these groups and important information about decomposition numbers.

It is natural to ask what features of the classical Schur algebras have $q$ analogues. In this paper, we define $q$-analogues of tensor space, of Weyl modules, and of weight spaces, thereby generalizing the main results which appear in Green's book [5]. For example, we classify the irreducible modules for $q$ Schur algebras, we determine bases for $q$-Weyl modules compatible with weight spaces, and we give results on composition multiplicities of the irreducible modules in $q$-Weyl modules. The proofs are largely self-contained, so by specializing $q$ to 1 , we recover the corresponding results in [5]. This paper, therefore, is relevant to the representation theory of symmetric groups and to the representation theory of general linear groups in the describing and in the nondescribing characteristics.

\section{The $q$-SChUR ALGEBRA}

Let $r$ be a natural number, let $R$ be an integral domain, and let $q$ be a unit in $R$. We denote the symmetric group on $r$ letters by $\mathfrak{S}_{r}$. The Hecke algebra $\mathscr{H}$ is the $R$-free $R$-algebra with basis $\left\{T_{w} \mid w \in \mathfrak{S}_{r}\right\}$, where the multiplication is determined by the following rule. If $a=(i, i+1)$ is a basic transposition in

Received by the editors August 15, 1989.

1980 Mathematics Subject Classification (1985 Revision). Primary 16A64, 16A65; Secondary $20 \mathrm{C} 30$.

This research was supported in part by NSF Grant No. DMS-8802290. The authors gratefully acknowledge support received from NATO Grant No. 0222/87. 
$\mathfrak{S}_{r}$ and $w \in \mathfrak{S}_{r}$ then

$$
T_{w} T_{a}= \begin{cases}T_{w a} & \text { if } l(w a)>l(w), \\ q T_{w a}+(q-1) T_{w} & \text { otherwise. }\end{cases}
$$

Here, $l(u)$ is the length of $u \in \mathfrak{S}_{r}$.

We shall be concerned with certain ideals of $\mathscr{H}$ which are indexed by compositions. A composition $\lambda=\left(\lambda_{1}, \lambda_{2}, \ldots, \lambda_{n}\right)$ of $r$ (written $\lambda \vDash r$ ) is a finite sequence of nonnegative integers whose sum is $r$. If the sequence $\lambda$ is nonincreasing then we call $\lambda$ a partition of $r$ and write $\lambda \vdash r$. If $\lambda=\left(\lambda_{1}, \lambda_{2}, \ldots\right) \models r$ then we define $\lambda^{\prime}=\left(\lambda_{1}^{\prime}, \lambda_{2}^{\prime}, \ldots\right)$ by setting $\lambda_{i}^{\prime}$ equal to the cardinality of $\left\{\lambda_{j} \mid \lambda_{j} \geq i\right\}$. Note that $\lambda^{\prime}$ is a partition of $r$; it is called the partition conjugate to $\lambda$. For $\lambda$ and $\mu$ compositions of $r$, we say that $\lambda$ and $\mu$ are associated if $\lambda^{\prime}=\mu^{\prime}$; that is, if $\mu$ can be derived from $\lambda$ by reordering the parts of $\lambda$.

If $\lambda=\left(\lambda_{1}, \lambda_{2}, \ldots\right)$ is a composition of $r$, then the corresponding standard Young subgroup $\mathfrak{S}_{\lambda}$ of $\mathfrak{S}_{r}$ consists of those permutations of $\{1,2, \ldots, r\}$ which leave invariant the following sets of integers: $\left\{1,2, \ldots, \lambda_{1}\right\},\left\{\lambda_{1}+\right.$ $\left.1, \lambda_{1}+2, \ldots, \lambda_{1}+\lambda_{2}\right\},\left\{\lambda_{1}+\lambda_{2}+1, \ldots\right\}, \ldots$ Let $\lambda$ and $\mu$ be compositions of $r$. In each coset of $\mathfrak{S}_{\lambda}$ in $\mathfrak{S}_{r}$, there is a unique element of minimal length, the distinguished coset representative. The set of distinguished coset representatives for right cosets $\mathfrak{S}_{\lambda} w$ is denoted by $\mathscr{D}_{\lambda}$. (Our convention for composing permutations $u$ and $v$ is that $u v$ denotes $u$ followed by $v$.) The set of distinguished left coset representatives is $\mathscr{D}_{\lambda}^{-1}$ and $\mathscr{D}_{\lambda} \cap \mathscr{D}_{\mu}^{-1}$ is the set of distinguished $\mathfrak{S}_{\lambda}-\mathfrak{S}_{\mu}$ double coset representatives. Let $\mathscr{D}_{\lambda \mu}=\mathscr{D}_{\lambda} \cap \mathscr{D}_{\mu}^{-1}$.

We shall consider the right ideals $x_{\lambda} \mathscr{H}$ of $\mathscr{H}$, where $x_{\lambda}=\sum_{w \in \mathfrak{S}_{i}} T_{w}$. The following holds.

(1.1) If $\lambda^{\prime}=\mu^{\prime}$ then $d^{-1} \mathfrak{S}_{\lambda} d=\mathfrak{S}_{\mu}$ for some $d \in \mathscr{D}_{\lambda \mu}$. Hence $x_{\lambda} T_{d}=T_{d} x_{\mu}$ and the right ideals $x_{\lambda} \mathscr{H}$ and $x_{\mu} \mathscr{H}$ are isomorphic.

We are going to define the $q$-Schur algebra to be the endomorphism algebra of an external direct sum of right ideals $x_{\lambda} \mathscr{H}$. Whether we take the direct sum to be over all partitions $\lambda$ of $r$ or over compositions $\lambda$ of $r$ usually makes little difference, in view of result (1.1). However, it is convenient to deal with restricted compositions. We therefore define $\Lambda(n, r)$ to be the set of compositions $\lambda=\left(\lambda_{1}, \lambda_{2}, \ldots, \lambda_{n}\right)$ of $r$ into $n$ parts (each part $\lambda_{i}$ being nonnegative).

1.2. Definition. Let $S_{R}(n, r)=\operatorname{End}_{\mathscr{H}}\left(\bigoplus_{\lambda \in \Lambda(n, r)} x_{\lambda} \mathscr{H}\right)$. We call $S_{R}(n, r)$ the $q$-Schur algebra.

When $q=1, \mathscr{H}$ is isomorphic to $\mathfrak{S}_{r}$, so $S_{R}(n, r)$ is the usual Schur algebra, as defined in Green's book [5]. Note that when $n \geq r$, every partition of $r$ is in $\Lambda(n, r)$, so by remark (1.1), we have

1.3. Lemma. If $n \geq r$ then the $q$-Schur algebra $S_{R}(n, r)$ is Morita equivalent to $\operatorname{End}_{\mathscr{H}}\left(\bigoplus_{\lambda \vdash r} x_{\lambda} \mathscr{H}\right)$. 
In [4], we took the $q$-Schur algebra to be defined in terms of partitions, and Lemma 1.3 enables us to translate the results in this paper directly into the alternative version of the $q$-Schur algebra.

If $\lambda$ and $\mu$ are compositions of $r$ and $d \in \mathfrak{S}_{r}$ then we obtain an element $\phi_{\lambda \mu}^{d}$ of $\operatorname{Hom}_{\mathscr{H}}\left(x_{\mu} \mathscr{H}, x_{\lambda} \mathscr{H}\right)$ by defining $\phi_{\lambda \mu}^{d}\left(x_{\mu} h\right)=\sum_{w \in \mathfrak{S}_{\lambda} d \mathfrak{S}_{\mu}} T_{w} h$ for $h \in \mathscr{H}$. Since the $q$-Schur algebra is a direct sum of spaces of the form $\operatorname{Hom}_{\mathscr{H}}\left(x_{\mu} \mathscr{H}, x_{\lambda} \mathscr{H}\right)$, these homomorphisms $\phi_{\lambda \mu}^{d}$ give us elements of the $q$ Schur algebra, by extending $\phi_{\lambda \mu}^{d}$ in the natural way, and we denote this element of the $q$-Schur algebra also by $\phi_{\lambda \mu}^{d}$. The following straightforward result was proved in $[2,3.4]$.

1.4. Theorem. The q-Schur algebra $S_{R}(n, r)$ is free as an $R$-module, with basis $\left\{\phi_{\lambda \mu}^{d} \mid \lambda, \mu \in \Lambda(n, r), d \in \mathscr{D}_{\lambda \mu}\right\}$.

In the case where $q=1$, our basis element $\phi_{\lambda \mu}^{d}$ coincides with a certain term $\xi_{i j}$ which is described in [5, §2.3]. Although it is possible to give a concise formula for the product of two basis elements when $q=1[5,(2.3 \mathrm{~b})]$ the product when $q$ is arbitrary seems to be extremely unpleasant. Before saying more about the product, we shall construct an antiautomorphism of the $q$-Schur algebra. This will turn up as a special case of the following general construction.

For the moment, let $\mathscr{H}$ be an arbitrary $R$-free $R$-algebra and suppose that we have an antiautomorphism $h \mapsto h^{*}$ of $\mathscr{H}$ which satisfies $h^{* *}=h$ for all $h \in$ $\mathscr{H}$. Let $M$ be a finite-dimensional right $\mathscr{H}$-lattice. Define the contravariant dual right $\mathscr{H}$-lattic $M^{*}$ in the usual way: $M^{*}=\operatorname{Hom}_{R}(M, R)$, where $\mathscr{H}$ acts by $(f h) m=f\left(m h^{*}\right)$ for $f \in M^{*}, h \in \mathscr{H}$, and $m \in M$. Let $\operatorname{End}_{\mathscr{H}}(M)$ be the ring of $\mathscr{H}$-endomorphisms of $M$, acting on the left. For $s \in \operatorname{End}_{\mathscr{H}}(M)$, define $s^{\#} \in \operatorname{End}_{\mathscr{L}}\left(M^{*}\right)$ by the rule $s^{\#} f=f \circ s$ for $f \in M^{*}$. Then $s \mapsto s^{\#}$ is an anti-isomorphism from $\operatorname{End}_{\mathscr{H}}(M)$ to $\operatorname{End}_{\mathscr{H}}\left(M^{*}\right)$. (With respect to the dual basis of $M$ and $M^{*}, s \mapsto s^{*}$ corresponds to transposing matrices.)

Now assume that $M$ is self-dual. Thus, there exists an $\mathscr{H}$-isomorphism $\theta$ from $M$ to $M^{*}$, and this induces an isomorphism $\hat{\theta}$ from $\operatorname{End}_{\mathscr{H}}(M)$ to $\operatorname{End}_{\mathscr{C}}\left(M^{*}\right)$. Applying first ${ }^{\#}$ and then $\hat{\theta}^{-1}$ we get an antiautomorphism ${ }^{*}$ of $\operatorname{End}_{\mathscr{H}}(M)$. Thus, we have

$$
\begin{aligned}
s^{*}(m) & =\hat{\theta}^{-1} s^{\#}(m)=\theta^{-1} s^{\#} \theta(m) \\
& =\theta^{-1}(\theta(m) \circ s) \quad \text { for all } s \in \operatorname{End}_{\mathscr{H}}(M) \text { and } m \in M .
\end{aligned}
$$

Of course, $\theta(m) n=\langle m, n\rangle$ gives a well-known correspondence between the set of $\mathscr{l}$-isomorphisms $\theta$ from $M$ to $M^{*}$ and the set of nondegenerate bilinear forms $\langle$,$\rangle on M$ which have the property that $\langle m h, n\rangle=\left\langle m, n h^{*}\right\rangle$ for all $m, n \in M$ and $h \in \mathscr{H}$. In this situation, we have $\left\langle s^{*} m, n\right\rangle=\theta\left(s^{*} m\right) n=$ $\left(\theta\left(\theta^{-1} s^{\#} \theta m\right)\right) n=s^{\#}(\theta m) n=(\theta m)(s n)=\langle m, s n\rangle$. Thus, we have

$$
\left\langle s^{*} m, n\right\rangle=\langle m, s n\rangle \quad \text { for all } s \in \operatorname{End}_{\mathscr{H}}(M) \text { and } m, n \in M .
$$


We note that this implies that not only is $M$ self-dual as a right $\mathscr{H}$-module (by hypothesis), but $M$ is also self-dual as a left $\operatorname{End}_{\mathscr{H}}(M)$-module.

Now let $\mathscr{H}$ again be the Hecke algebra, and let $M=\bigoplus_{\lambda \in \Lambda(n, r)} x_{\lambda} \mathscr{H}$. For $\lambda \in \Lambda(n, r)$, we define a (symmetric) bilinear form $\langle,\rangle_{\lambda}$ on $x_{\lambda} \mathscr{H}$ by setting

$$
\left\langle x_{\lambda} T_{u}, x_{\lambda} T_{v}\right\rangle_{\lambda}= \begin{cases}q^{l(u)} & \text { if } u=v, \\ 0 & \text { otherwise }\end{cases}
$$

for $u, v \in \mathscr{D}_{\lambda}$. We extend these bilinear forms to a nondegenerate bilinear form $\langle$,$\rangle on M$, by specifying that $x_{\lambda} \mathscr{H}$ and $x_{\mu} \mathscr{H}$ are orthogonal if $\lambda \neq \mu$.

It is known that $\mathscr{H}$ has an antiautomorphism ${ }^{*}$ which is defined by $T_{w}^{*}=$ $T_{w^{-1}}$ for $w \in \mathfrak{S}_{r}$, and that the bilinear form on $M$ satisfies

$$
\langle m h, n\rangle=\left\langle m, n h^{*}\right\rangle \text { for all } m, n \in M \text { and } h \in \mathscr{H} \text {. }
$$

Let $\omega$ denote the partition $\left(1^{r}\right)=(1,1, \ldots, 1)$ of $r$. Note that $x_{\omega} \mathscr{H}=\mathscr{H}$ and

$$
\left\langle h_{1} h_{2}, h_{3}\right\rangle_{\omega}=\left\langle h_{2}, h_{1}^{*} h_{3}\right\rangle_{\omega}=\left\langle h_{1}, h_{3} h_{2}^{*}\right\rangle_{\omega} \text { for all } h_{1}, h_{2}, h_{3} \in \mathscr{H} .
$$

Consequently,

$$
\left\langle h_{1}, h_{2}\right\rangle_{\omega}=\left\langle h_{1}^{*}, h_{2}^{*}\right\rangle_{\omega} \text { for all } h_{1}, h_{2} \in \mathscr{H} .
$$

The results (1.8) and (1.9) are proved in [2, 2.2 and 4.4].

Now, result (1.8) shows that $M$ is a self-dual $\mathscr{H}$-module, so by our previous discussion, there exists an antiautomorphism ${ }^{*}$ of the $q$-Schur algebra, $\operatorname{End}_{\mathscr{H}}(M)$. We next determine ${ }^{*}$ explicitly.

1.11. Theorem. Let $\lambda, \mu \in \Lambda(n, r)$ and $d \in \mathscr{D}_{\lambda \mu}$. Then the antiautomorphism * of $S_{R}(n, r)$ satisfies $\left(\phi_{\lambda \mu}^{d}\right)^{*}=\phi_{\mu \lambda}^{d^{-1}}$.

Proof. Suppose that $u$ and $v \in \mathfrak{S}_{r}$. Then, using results (1.8)-(1.10), we have

$$
\begin{aligned}
\left\langle x_{\lambda} T_{u}, \phi_{\lambda \mu}^{d}\left(x_{\mu} T_{v}\right)\right\rangle_{\lambda}=\left\langle x_{\lambda}, \sum_{w \in \mathfrak{S}_{\lambda} d \mathfrak{S}_{\mu}} T_{w} T_{v} T_{u^{-1}}\right\rangle_{\lambda} \\
=\text { the coefficient of } T_{1} \text { in } \sum_{w \in \mathfrak{S}_{\lambda} d \mathfrak{G}_{\mu}} T_{w} T_{v} T_{u^{-1}} \\
=\left\langle T_{1}, \sum_{w \in \mathfrak{S}_{i} d \mathfrak{G}_{\mu}} T_{w} T_{v} T_{u^{-1}}\right\rangle_{\omega}=\left\langle T_{u} T_{v^{-1}}, \sum_{w \in \mathfrak{S}_{\lambda} d \mathfrak{G}_{\mu}} T_{w}\right\rangle_{\omega} \\
=\left\langle T_{v} T_{u^{-1}}, \sum_{w \in \mathfrak{S}_{\mu} d^{-1} \mathfrak{S}_{i}} T_{w}\right\rangle_{\omega}=\left\langle T_{1}, \sum_{w \in \mathfrak{S}_{\mu} d^{-1} \mathfrak{S}_{\lambda}} T_{w} T_{u} T_{v^{-1}}\right\rangle_{\omega} \\
=\text { the coefficient of } T_{1} \text { in } \sum_{w \in \mathfrak{S}_{\mu} d^{-1} \mathfrak{S}_{i}} T_{w} T_{u} T_{v^{-1}} \\
=\left\langle\sum_{w \in \mathfrak{G}_{\mu} d^{-1} \mathfrak{S}_{i}} T_{w} T_{u} T_{v^{-1}}, x_{\mu}\right\rangle_{\mu}=\left\langle\phi_{\mu \lambda}^{d^{-1}}\left(x_{\lambda} T_{u}\right), x_{\mu} T_{v}\right\rangle_{\mu} .
\end{aligned}
$$


Hence $\left\langle m, \phi_{\lambda \mu}^{d} n\right\rangle=\left\langle\phi_{\mu \lambda}^{d^{-1}} m, n\right\rangle$ for all $m, n \in M$. But $\left\langle\phi_{\lambda \mu}^{d^{*}} m, n\right\rangle=$ $\left\langle m, \phi_{\lambda \mu}^{d} n\right\rangle$, by result (1.6), and it follows that $\left(\phi_{\lambda \mu}^{d}\right)^{*}=\phi_{\mu \lambda}^{d^{-1}}$.

In the case where $q=1$, Green denotes the antiautomorphism ${ }^{*}$ of the Schur algebra by $J[5, \S 2.7]$.

Because the $q$-Schur algebra has an antiautomorphism, we may construct from any $S_{R}(n, r)$-module $M$ the contravariant dual module $M^{*}$ in the usual way. That is, $M^{*}$ is defined to be $\operatorname{Hom}_{R}(M, R)$ and $s \in S_{R}(n, r)$ acts on $f \in M^{*}$ by $(f s) m=f\left(m s^{*}\right)$ for all $m \in M$.

1.2. Remark. Every $\mathscr{H}$-homomorphism from $x_{\mu} \mathscr{H}$ to $x_{\lambda} \mathscr{H}$ is given by left multiplication by an element of $\mathscr{H}$. Right multiplication by the same element gives an $\mathscr{H}$-homomorphism from $\mathscr{H} x_{\lambda}$ to $\mathscr{H} x_{\mu}$. Mapping left multiplication by $h$ to right multiplication by $h$ gives an anti-isomorphism of $\operatorname{End}_{\mathscr{H}}\left(\bigoplus_{\lambda} x_{\lambda} \mathscr{H}\right)$ to $\operatorname{End}_{\mathscr{H}}\left(\bigoplus_{\lambda} \mathscr{H} x_{\lambda}\right)$. We may compose this anti-isomorphism with the antiautomorphism ${ }^{*}$ of $S_{R}(n, r)$ to get a canonical identification of the endomorphism rings $\operatorname{End}_{\mathscr{H}}\left(\bigoplus_{\lambda} x_{\lambda} \mathscr{H}\right)$ and $\operatorname{End}_{\mathscr{H}}\left(\bigoplus_{\lambda} \mathscr{H} x_{\lambda}\right)$. Under this identification, $\phi_{\lambda \mu}^{d}$ as an element of $\operatorname{End}_{\mathscr{H}}\left(\bigoplus_{\lambda} x_{\lambda} \mathscr{H}\right)$ sends $x_{\mu} h$ to $\sum_{w \in \mathfrak{S}_{\lambda} d \mathfrak{G}_{\mu}} T_{w} h$ and $\phi_{\lambda \mu}^{d}$ as an element of $\operatorname{End}_{\mathscr{H}}\left(\bigoplus \mathscr{H} x_{\lambda}\right)$ sends $h x_{\lambda}$ to $\sum_{w \in \mathfrak{S}_{\lambda} d \mathfrak{S}_{\mu}} h T_{w}^{\mu}$ for all $h \in \mathscr{H}$.

We shall often be using homomorphisms in this paper, and we always adopt the convention that the homomorphism is written on the side opposite to that of the ring action. Thus, for example, if $M$ is a right $\mathscr{H}$-module then the image of $m \in M$ under an $\mathscr{H}$-homomorphism $\phi$ is written as $\phi m$, but if $M$ is a left $\mathscr{H}$-module then we write $m \phi$.

\section{2. $q$-TENSOR SPACE}

Homogeneous polynomial representations of general linear groups can be described by the action of classical Schur algebras $S_{R}(n, r)$ (that is, the case where $R$ is a field and $q=1)$ on the tensor space $E^{\otimes r}=E \otimes E \otimes \cdots \otimes E(r$ times), where $E$ is the natural module for $G L_{n}(R)$ (see, for example, [5]). We shall set up a $q$-analogue of $E^{\otimes r}$.

First, we discuss products of the basis elements $\phi_{\lambda \mu}^{d}$ of the $q$-Schur algebra which we gave in Theorem 1.4. Let $\rho, \sigma, \lambda$, and $\mu$ be compositions of $r$, let $u \in \mathscr{D}_{\rho \sigma}$ and $d \in \mathscr{D}_{\lambda \mu}$. Recall that $\phi_{\lambda \mu}^{d}$ maps $x_{\mu} \mathscr{H}$ into $x_{\lambda} \mathscr{H}$ and $\phi_{\lambda \mu}^{d}$ annihilates $x_{\rho} \mathscr{H}$ if $\rho \neq \mu$. Therefore,

$$
\phi_{\rho \sigma}^{u} \phi_{\lambda \mu}^{d}=0 \text { unless } \sigma=\lambda .
$$

When $\sigma=\lambda$, the product $\phi_{\rho \sigma}^{u} \phi_{\lambda \mu}^{d}$ is a linear combination of terms $\phi_{\rho \mu}^{e}$ with $e \in \mathscr{D}_{\rho \mu}$, but we know of no formula for calculating the coefficients, in general.

Next, note that $\phi_{\lambda \lambda}^{1}$ acts as the identity on $x_{\lambda} \mathscr{H}$. Therefore,

$$
\phi_{\lambda \lambda}^{1} \phi_{\lambda \mu}^{d}=\phi_{\lambda \mu}^{d}=\phi_{\lambda \mu}^{d} \phi_{\mu \mu}^{1} \text {. }
$$


In particular,

$$
\phi_{\lambda \lambda}^{1} S_{R}(n, r) \phi_{\mu \mu}^{1} \cong \operatorname{Hom}_{\mathscr{H}}\left(x_{\mu} \mathscr{H}, x_{\lambda} \mathscr{H}\right)
$$

Also,

(2.4) $\left\{\phi_{\lambda \lambda}^{1} \mid \lambda \in \Lambda(n, r)\right\}$ is a set of orthogonal idempotents of $S_{R}(n, r)$ whose sum is the identity element of $S_{R}(n, r)$.

We now assume, until further notice, that $n \geq r$. In $\S 8$ we shall explain how to adapt several of our results to the case where $n<r$.

We fix $\omega$ to be the partition $\left(1^{r}\right)=(1,1, \ldots, 1)$ of $r$. Since $n \geq r$, we have $\omega \in \Lambda(n, r)$. Note that $x_{\omega}$ is the identity element $T_{1}$ of $\mathscr{H}$.

2.5. Lemma. Suppose that $s \in S_{R}(n, r)$ and $\lambda \in \Lambda(n, r)$. We have: $s \phi_{\lambda \lambda}^{1}=0$ if and only if $s \phi_{\lambda \omega}^{1}=0$.

Proof. If $s \phi_{\lambda \lambda}^{1}=0$ then $0=s \phi_{\lambda \lambda}^{1} \phi_{\lambda \omega}^{1}=s \phi_{\lambda \omega}^{1}$. Conversely, if $s \phi_{\lambda \omega}^{1}=0$ then $0=s \phi_{\lambda \omega}^{1}\left(x_{\omega} h\right)=s\left(x_{\lambda} h\right)=s \phi_{\lambda \lambda}^{1}\left(x_{\lambda} h\right)$ for all $h \in \mathscr{H}$, so $s \phi_{\lambda \lambda}^{1}=0$.

The lemma shows that post-multiplication by $\phi_{\lambda \omega}^{1}$ embeds the left ideal $S_{R}(n, r) \phi_{\lambda \lambda}^{1}$ in the left ideal $S_{R}(n, r) \phi_{\omega \omega}^{1}$. But the $q$-Schur algebra is a direct sum of left ideals $S_{R}(n, r) \phi_{\lambda \lambda}^{1}$ (see result $(2.4)$ ). Therefore, when $R$ is a field, every irreducible module for the $q$-Schur algebra occurs as a composition factor of the left ideal $S_{R}(n, r) \phi_{\omega \omega}^{1}$. This remark indicates why the set $S_{R}(n, r) \phi_{\omega \omega}^{1}$ is important.

2.6. Definition. We denote the subset $S_{R}(n, r) \phi_{\omega \omega}^{1}$ of the $q$-Schur algebra by $E_{R}(n, r)$, and we call it q-tensor space.

Now, $\phi_{\omega \omega}^{1} S_{R}(n, r) \phi_{\omega \omega}^{1}=\operatorname{Hom}_{\mathscr{H}}\left(x_{\omega} \mathscr{H}, x_{\omega} \mathscr{H}\right)=\operatorname{End}_{\mathscr{H}}(\mathscr{H})$, and $\operatorname{End}_{\mathscr{H}}(\mathscr{H})$ is canonically isomorphic to $\mathscr{H}$. We shall freely identify $\mathscr{H}$ with the subalgebra $\phi_{\omega \omega}^{1} S_{R}(n, r) \phi_{\omega \omega}^{1}$ of $S_{R}(n, r)$. (If $u \in \mathfrak{S}_{r}$ then the isomorphism between $\mathscr{H}$ and $\phi_{\omega \omega}^{1} S_{R}(n, r) \phi_{\omega \omega}^{1}$ sends $T_{u}$ to $\phi_{\omega \omega}^{u}$ and sends $T_{u}^{*}$ to $\left(\phi_{\omega \omega}^{u}\right)^{*}$.) Using this identification, we have:

(2.7) $q$-tensor space $E_{R}(n, r)$ is an $\left(S_{R}(n, r)-\mathscr{H}\right)$-bimodule.

Since $\phi_{\omega \omega}^{1}$ is an idempotent, $q$-tensor space is a free $R$-module. In view of results $(2.1)$ and $(2.2)$, we have

(2.8) $\left\{\phi_{\lambda \omega}^{d} \mid \lambda \in \Lambda(n, r), d \in \mathscr{D}_{\lambda}\right\}$ is a basis of the $R$-lattice $E_{R}(n, r)$. Moreover, $\phi_{\lambda \omega}^{d}=\phi_{\lambda \omega}^{1} T_{d}$.

It is helpful to understand why $E_{R}(n, r)$ is a $q$-analogue of tensor space, and we explain this now. Take $E$ to be a free $R$-module with basis $e_{1}, e_{2}, \ldots, e_{n}$. Then $\left\{e_{i_{1}} \otimes e_{i_{2}} \otimes \cdots \otimes e_{i_{r}} \mid i_{1}, i_{2}, \ldots, i_{r} \in\{1,2, \ldots, n\}\right\}$ is a basis of $E^{\otimes r}$. The symmetric group $\mathfrak{S}_{r}$ acts on $E^{\otimes r}$ on the right by place permutations. We define an $R$-isomorphism between $E_{R}(n, r)$ and $E^{\otimes r}$ by extending the following map 
to be linear:

$$
\phi_{\lambda \omega}^{d} \mapsto(\underbrace{e_{1} \otimes \cdots \otimes e_{1}}_{\lambda_{1} \text { times }} \otimes \underbrace{e_{2} \otimes \cdots \otimes e_{2}}_{\lambda_{2} \text { times }} \otimes \cdots \otimes \underbrace{e_{n} \otimes \cdots \otimes e_{n}}_{\lambda_{n} \text { times }}) d .
$$

It is easy to check, in the case where $q=1$, that this map is a bimodule isomorphism. When $q=1, \mathscr{H}$ is the group algebra of $\mathfrak{S}_{r}$ and $S_{R}(n, r)$ is the usual Schur algebra.

2.9. Definition. Suppose that $\mu \in \Lambda(n, r)$. Let $M^{\mu}$ be the right $\mathscr{H}$-submodule $\phi_{\mu \omega}^{1} \mathscr{H}$ of $q$-tensor space.

2.10. Theorem. We have $M^{\mu}=\operatorname{Hom}_{\mathscr{H}}\left(\mathscr{H}, x_{\mu} \mathscr{H}\right)=\phi_{\mu \mu}^{1} S_{R}(n, r) \phi_{\omega \omega}^{1}$. The right $\mathscr{H}$-modules $M^{\mu}$ and $x_{\mu} \mathscr{H}$ are isomorphic. $A$ basis $M^{\mu}$ is given by $\left\{\phi_{\mu \omega}^{d} \mid d \in \mathscr{D}_{\mu}\right\}$.

Proof. From (2.1) and (2.2), we see that both $\phi_{\mu \omega}^{1} \mathscr{H}$ and $\phi_{\mu \mu}^{1} S_{R}(n, r) \phi_{\omega \omega}^{1}$ have basis $\left\{\phi_{\mu \omega}^{d} \mid d \in \mathscr{D}_{\mu}\right\}$. Therefore, $M^{\mu}=\phi_{\mu \mu}^{1} S_{R}(n, r) \phi_{\omega \omega}^{1}$, which in turn is equal to $\operatorname{Hom}_{\mathscr{H}}\left(\mathscr{H}, x_{\mu} \mathscr{H}\right)$, by result (2.3). The isomorphism between $x_{\mu} \mathscr{H}$ and $M^{\mu}$ exists on general grounds; explicitly, it is given by $x_{\mu} h \mapsto \phi_{\mu \omega}^{1} h(h \in \mathscr{H})$.

The action of $\mathscr{H}$ on our basis of $M^{\mu}$ was given in [2, 2.3ii)]:

(2.11) If $a=(i, i+1)$ is a basic transposition in $\mathfrak{S}_{r}$ and $d \in \mathscr{D}_{\mu}$ then

$$
\phi_{\mu \omega}^{d} T_{a}= \begin{cases}q \phi_{\mu \omega}^{d} & \text { if } l(d a)=l(d)+1 \text { and } d a \notin \mathscr{D}_{\mu}, \\ \phi_{\mu \omega}^{d a} & \text { if } l(d a)=l(d)+1 \text { and } d a \in \mathscr{D}_{\mu} \\ q \phi_{\mu \omega}^{d a}+(q-1) \phi_{\mu \omega}^{d} & \text { if } l(d a)=l(d)-1 .\end{cases}
$$

If $\lambda$ and $\mu$ are associated compositions, then $x_{\lambda} \mathscr{H}$ and $x_{\mu} \mathscr{H}$ are isomorphic, in view of result (1.1), and therefore $M^{\lambda}$ and $M^{\mu}$ are isomorphic. Furthermore, by multiplying $E_{R}(n, r)$ on the left by $\sum_{\lambda \in \Lambda(n, r)} \phi_{\lambda \lambda}^{1}$, which is the identity of $S_{R}(n, r)$, we obtain

$$
E_{R}(n, r)=\bigoplus_{\lambda \in \Lambda(n, r)} M^{\lambda} \text { as a right } \mathscr{H} \text {-module. }
$$

2.13. Definition. Let $U$ be a left $S_{R}(n, r)$-module and let $\lambda \in \Lambda(n, r)$. Then the $R$-submodule $\phi_{\lambda \lambda}^{1} U$ of $U$ is the $q$-weight space of $U$ which corresponds to the weight $\lambda$.

Note that $\phi_{\lambda \lambda}^{1} U$ is $R$-free if $U$ is $R$-free. We have $U=\bigoplus_{\lambda \in \Lambda(n, r)} \phi_{\lambda \lambda}^{1} U$, since the elements $\phi_{\lambda \lambda}^{1}$ are orthogonal idempotents of $S_{R}(n, r)$ whose sum is 1 . This decomposition of $U$ is called the q-weight decomposition of $U$. (Compare $[5, \S 3]$.) For example, result $(2.12)$ gives us the $q$-weight decomposition of $q$ tensor space. The following result is obvious. 
2.14. Theorem. Suppose that $\widetilde{U} \leq U$ are $S_{R}(n, r)$-modules. Then each $q$ weight space of $\widetilde{U}$ is the intersection with $\widetilde{U}$ of the corresponding q-weight space of $U$. In particular, if $\widetilde{U}$ is an $S_{R}(n, r)$-submodule of q-tensor space, then $\widetilde{U}=\bigoplus_{\lambda \in \Lambda(n, r)}\left(\widetilde{U} \cap M^{\lambda}\right)$ as an R-module.

\section{3. $q$-SPECHT MODUles AND $q$-WeYl MODUles}

If $\lambda \vDash r$, then we define $y_{\lambda} \in \mathscr{H}$ by

$$
y_{\lambda}=\sum_{w \in \mathfrak{G}_{i}}(-q)^{-l(w)} T_{w} .
$$

Because there is an outer automorphism of $\mathscr{H}$ which sends $x_{\lambda}$ to $y_{\lambda}$, it is possible to interchange the roles of $x_{\lambda}$ and $y_{\lambda}$ in many places. For example, the $q$-Schur algebra, which we defined to be $\operatorname{End}_{\mathscr{H}}\left(\bigoplus_{\lambda \in \Lambda(n, r)} x_{\lambda} \mathscr{H}\right)$, turns out to be isomorphic to $\operatorname{End}_{\mathscr{H}}\left(\bigoplus_{\lambda \in \Lambda(n, r)} y_{\lambda} \mathscr{H}\right)$. Details can be found in [4].

Properties of the subsets $y_{\lambda} \mathscr{H} x_{\mu}$ of $\mathscr{H}$ are of great importance, and we recall these now. We define the relation $\unrhd$ on $\Lambda(n, r)$ by writing $\lambda \unrhd \mu$ if $\sum_{i=1}^{j} \lambda_{i}^{\prime} \leq \sum_{i=1}^{j} \mu_{i}^{\prime}$ for all $j$. Thus, the restriction of $\unrhd$ to the set of partitions of $r$ is the usual dominance order, and the results $\lambda \unrhd \mu$ and $\mu \unrhd \lambda$ hold simultaneously if and only if $\lambda$ and $\mu$ are associated compositions. We write $\lambda \triangleright \mu$ if $\lambda \unrhd \mu$ and $\sum_{i=1}^{j} \lambda_{i}^{\prime}<\sum_{i=1}^{j} \mu_{i}^{\prime}$ for some $j$. For $\lambda \vDash r$, the element $w_{\lambda}$ of $\mathscr{D}_{\lambda \lambda^{\prime}}$ is defined to be the unique $\mathfrak{S}_{\lambda}-\mathfrak{S}_{\lambda^{\prime}}$ double coset representative with the trivial intersection property; that is, $w_{\lambda}^{-1} \mathfrak{S}_{\lambda} w_{\lambda} \cap \mathfrak{S}_{\lambda^{\prime}}=(1)$. The following results were proved in $[2,4.1]$ :

(3.1) Suppose that $\lambda, \mu \models r$. Then

(i) $x_{\lambda} \mathscr{H} y_{\mu} \neq(0)$ only if $\mu^{\prime} \unrhd \lambda$,

(ii) $x_{\lambda} \mathscr{H} y_{\lambda^{\prime}}$ is a free $R$-module of rank one, spanned by $x_{\lambda} T_{w_{\lambda}} y_{\lambda^{\prime}}$.

The next lemma translates these results into statements about the $q$-Schur algebra.

3.2. Lemma. Suppose that $\lambda, \mu \in \Lambda(n, r)$. Then

(i) $\phi_{\lambda \lambda}^{1} S_{R}(n, r) y_{\mu} \neq 0$ only if $\mu^{\prime} \unrhd \lambda$,

(ii) $\phi_{\lambda \lambda}^{1} S_{R}(n, r) y_{\lambda^{\prime}}$ is a free $R$-module of rank one, spanned by $\phi_{\lambda \omega}^{1} T_{w_{\lambda}} y_{\lambda^{\prime}}$.

Proof. Note that $\phi_{\lambda \lambda}^{1} S_{R}(n, r) y_{\mu} \subseteq \phi_{\lambda \lambda}^{1} S_{R}(n, r) \phi_{\omega \omega}^{1}=M^{\lambda}$. We saw in the proof of Theorem 2.10 how $x_{\lambda} h \mapsto \phi_{\lambda \omega}^{1} h$ gives an isomorphism between the right $\mathscr{H}$-modules $x_{\lambda} \mathscr{H}$ and $M^{\lambda}$, and this enables us to deduce the results in the lemma from the results in (3.1).

3.3. Definition. Suppose that $\lambda \in \Lambda(n, r)$. Let $z_{\lambda}=\phi_{\lambda \omega}^{1} T_{w_{\lambda}} y_{\lambda^{\prime}}$, and let $S^{\lambda}$ be the $\mathscr{H}$-submodule $z_{\lambda} \mathscr{H}$ of $M^{\lambda}$. We call $S^{\lambda}$ a $q$-Specht module.

Under the canonical isomorphism $x_{\lambda} h \mapsto \phi_{\lambda \omega}^{1} h$ between $x_{\lambda} \mathscr{H}$ and $M^{\lambda}$, $x_{\lambda} T_{w_{i}} y_{\lambda^{\prime}}$ is mapped to $z_{\lambda}$, so $S^{\lambda}$ is canonically isomorphic to the Specht mod- 
ule which was described in [2, §4]. By [2, 5.9] each $q$-Specht module is free as an $R$-module. In $[2,4.3]$ we proved that $S^{\lambda}$ and $S^{\mu}$ are isomorphic if the compositions $\lambda$ and $\mu$ are associated.

Now suppose that $\lambda, \mu \in \Lambda(n, r)$ and $\mu \nsubseteq \lambda$. Then, for all $d \in \mathscr{D}_{\lambda \mu}$, we have $\phi_{\lambda \mu}^{d} S^{\mu}=\phi_{\lambda \mu}^{d} \phi_{\mu \omega}^{1} T_{w_{\mu}} y_{\mu^{\prime}} \mathscr{L} \subseteq \phi_{\lambda \lambda}^{1} S_{R}(n, r) y_{\mu^{\prime}} \mathscr{H}=0$, by Lemma 3.2(i). Therefore, we have the following result, which, in the case where $q=1$, is well known and fundamental to the representation theory of the symmetric group.

(3.4) If $\phi \in \operatorname{Hom}_{\lambda}\left(M^{\mu}, M^{\lambda}\right)$ and $\mu \nsubseteq \lambda$ then $\phi S^{\mu}=(0)$.

A very important property of the $q$-Specht module $S^{\mu}$ is that it is equal to the intersection of all the kernels of homomorphisms of the kind described in result (3.4); indeed, $S^{\mu}$ equals the intersection of a certain subset of such kernels. In the case where $R$ is a field, this result was described in [2, 7.5]. We wish to restate the theorem now, and extend it to the case where $R$ is an integral domain.

3.5. Definition. For the moment, fix $\mu=\left(\mu_{1}, \mu_{2}, \ldots, \mu_{n}\right)$ to be a partition of $r$. If $1 \leq i \leq n-1$ and $0 \leq j \leq \mu_{i+1}$, then let $\psi_{i j}=\phi_{\lambda \mu}^{1}$, where $\lambda=\left(\lambda_{1}, \lambda_{2}, \ldots, \lambda_{n}\right)$ is the composition of $r$ for which $\lambda_{i}=\mu_{i}+\mu_{i+1}-j$, $\lambda_{i+1}=j$, and $\lambda_{a}=\mu_{a}$ if $1 \leq a \leq n$ and $a \neq i$ or $i+1$. Note that $\lambda \unrhd \mu$.

3.6. Theorem (the Kernel Intersection Theorem for $q$-Specht modules). Suppose that $\mu \vdash r$ and that $\mu$ has precisely $k$ nonzero parts. Then

$$
S^{\mu}=\bigcap_{i=2}^{k} \bigcap_{j=0}^{\mu_{i}-1} \operatorname{ker} \psi_{i-1, j} .
$$

Proof. The theorem is true when $R$ is a field by [2, 7.5]. When $R$ is an integral domain, let $v$ be in the kernel intersection. Since $v \in \phi_{\mu \omega}^{1} \mathscr{H}$, we may regard $v$ as an element of $\phi_{\mu \omega}^{1} \mathscr{H}_{F}$, where $F$ denotes the quotient field of $R$, and $\mathscr{H}_{F}$ denotes the Hecke algebra defined over $F$. Then $v$ belongs to $\phi_{\mu \omega}^{1} T_{w_{\mu}} y_{\mu^{\prime}} \mathscr{H}_{F}$, that is, the Specht module over $F$. Thus, $v \in \phi_{\mu \omega}^{1} \mathscr{H} \cap$ $\phi_{\mu \omega}^{1} T_{w_{\mu}} y_{\mu^{\prime}} \mathscr{H}_{F}=\phi_{\mu \omega}^{1} T_{w_{\mu}} y_{\mu^{\prime}} \mathscr{H}$, where the equality holds because of the Standard Basis Theorem [2, 5.6]. That is, $v \in S^{\mu}$, as we wished to show.

3.7. Corollary. Suppose that $\mu \in \Lambda(n, r)$. Then

$$
S^{\mu}=\left\{v \in M^{\mu} \mid \phi_{\lambda \mu}^{d} v=0 \text { for all } \lambda \in \Lambda(n, r) \text { with } \mu \notin \lambda \text { and all } d \in \mathscr{D}_{\lambda \mu}\right\} \text {. }
$$

We now define a left $S_{R}(n, r)$-module $L^{\mu}$ which has properties similar to those of the right $\mathscr{H}$-module $M^{\mu}$. Inside $L^{\mu}$ will be a $q$-Weyl module, which behaves in some ways like a $q$-Specht module.

3.8. Definitions. Suppose that $\mu \in \Lambda(n, r)$.

(i) Let $L^{\mu}$ be the left ideal $S_{R}(n, r) y_{\mu}$ of $S_{R}(n, r)$.

(ii) Let $W^{\mu}$ be the $S_{R}(n, r)$-submodule $S_{R}(n, r) z_{\mu}$ of $L^{\mu^{\prime}}$. We call $W^{\mu}$ a $q$-Weyl module. 
Note that we have $L^{\mu}=E_{R}(n, r) y_{\mu}$, since $y_{\mu}=\phi_{\omega \omega}^{1} y_{\mu}$. We have now introduced the four main modules which we shall use, so for the convenience of the reader, we gather their definitions together.

Summary. Suppose that $\mu \in \Lambda(n, r)$.

(i) $M^{\mu}$ is the right $\mathscr{H}$-submodule $\phi_{\mu \omega}^{1} \mathscr{H}$ of $q$-tensor space.

(ii) The $q$-Specht module $S^{\mu}$ is the right $\mathscr{H}$-submodule $z_{\mu} \mathscr{H}$ of $M^{\mu}$, where $z_{\mu}=\phi_{\mu \omega}^{1} T_{w_{\mu}} y_{\mu^{\prime}}$.

(iii) $L^{\mu}$ is the left $S_{R}(n, r)$-submodule $S_{R}(n, r) y_{\mu}$ of $q$-tensor space.

(iv) The $q$-Weyl module $W^{\mu}$ is the left $S_{R}(n, r)$-submodule $S_{R}(n, r) z_{\mu}$ of $L^{\mu^{\prime}}$.

3.9. Lemma. Suppose that $\lambda$ and $\mu$ are associated compositions in $\Lambda(n, r)$. Then $L^{\lambda} \cong L^{\mu}$ and $W^{\lambda}=W^{\mu}$.

Proof. For some $d \in \mathscr{D}_{\lambda \mu}$, we have $d^{-1} \mathfrak{S}_{\lambda} d=\mathfrak{S}_{\mu}$. Hence $y_{\lambda} T_{d}=T_{d} y_{\mu}$, and post-multiplication by $T_{d}$ gives an isomorphism from $L^{\lambda}$ to $L^{\mu}$. Also, $\phi_{\lambda \mu}^{d}$ is invertible. Now, $\phi_{\lambda \mu}^{d} z_{\mu}=\phi_{\lambda \mu}^{d} \phi_{\mu \omega}^{1} T_{w_{\mu}} y_{\mu^{\prime}} \in \phi_{\lambda \lambda}^{1} S_{R}(n, r) y_{\lambda^{\prime}}$ (here, we have used the fact that $\left.\lambda^{\prime}=\mu^{\prime}\right)$. Therefore, $\phi_{\lambda \mu}^{d} z_{\mu}=c \phi_{\lambda \omega}^{1} T_{w_{\lambda}} y_{\lambda^{\prime}}=c z_{\lambda}$ for some $c \in R$, by Lemma 3.2(ii). Thus, $c z_{\lambda} \in W^{\mu}$ and since $\phi_{\lambda \mu}^{d}$ is invertible, we deduce that $W^{\lambda}=W^{\mu}$.

\section{The IRREDUCIBLE $S_{R}(n, r)$-MOdULES $F^{\mu}$}

The irreducible $\mathscr{H}$-modules, when $R$ is a field, were constructed as top factors of certain $q$-Specht modules in [2], using a certain bilinear form on $M^{\mu}$. In a similar way, we are going to produce the irreducible $S_{R}(n, r)$-modules, when $R$ is a field, as top factors of $q$-Weyl modules.

We have seen in (2.12) how to identify $q$-tensor space with $\bigoplus_{\lambda \in \Lambda(n, r)} x_{\lambda} \mathscr{H}$. Therefore, there is a nondegenerate bilinear form $\langle$,$\rangle on q$-tensor space which satisfies the following (see results (1.6) and (1.8)):

(4.1) Let $u, v \in E_{R}(n, r), h \in \mathscr{H}$, and $s \in S_{R}(n, r)$. Then

(i) $\left\langle s^{*} u, v\right\rangle=\langle u, s v\rangle$,

(ii) $\langle u h, v\rangle=\left\langle u, v h^{*}\right\rangle$.

For $q=1$, the form $\langle$,$\rangle is the canonical form on tensor space [5, p. 33,$ Example 1 and $(5.1 \mathrm{c})]$. As in $[5, \S 5.5]$ we contract the form which is given by restricting $\langle$,$\rangle to L^{\mu}$.

4.2. Definition. Suppose that $\mu \in \Lambda(n, r)$. Define a bilinear form $\langle\langle\rangle$,$\rangle on$ $L^{\mu}=E_{R}(n, r) y_{\mu}$ by $\left\langle\left\langle u y_{\mu}, v y_{\mu}\right\rangle\right\rangle=\left\langle u, v y_{\mu}\right\rangle$ for $u, v \in E_{R}(n, r)$.

Note that $y_{\mu}^{*}=y_{\mu}$, so if $u y_{\mu}=u^{\prime} y_{\mu}$, where $u, u^{\prime} \in E_{R}(n, r)$, then

$$
\left\langle\left\langle u y_{\mu}, v y_{\mu}\right\rangle\right\rangle=\left\langle u, v y_{\mu}\right\rangle=\left\langle u y_{\mu}, v\right\rangle=\left\langle u^{\prime} y_{\mu}, v\right\rangle=\left\langle u^{\prime}, v y_{\mu}\right\rangle=\left\langle\left\langle u^{\prime} y_{\mu}, v y_{\mu}\right\rangle\right\rangle \text {. }
$$


This verifies that $\langle\langle\rangle$,$\rangle is well defined (compare [5,5.5 b]$ ).

Obviously, the contracted form inherits property $(4.1)(\mathrm{i})$ :

(4.3) Let $u, v \in L^{\mu}$ and $s \in S_{R}(n, r)$. Then $\left\langle\left\langle s^{*} u, v\right\rangle\right\rangle=\langle\langle u, s v\rangle\rangle$.

As usual, if $U$ is a subset of $L^{\mu}$ then $U^{\perp}$ denotes $\left\{v \in L^{\mu} \mid\langle\langle v, u\rangle\rangle=0\right.$ for all $u \in U\}$.

4.4. Theorem. Suppose that $R$ is a field and $\mu \in \Lambda(n, r)$. Let $U$ be an $S_{R}(n, r)$-submodule of $L^{\mu^{\prime}}$. Then $W^{\mu} \subseteq U$ or $U \subseteq W^{\mu \perp}$.

Proof (compare [2, 4.8]). If $\phi_{\mu \mu}^{1} U \neq 0$ then by Lemma 3.2(ii), $\phi_{\mu \omega}^{1} T_{w_{\mu}} y_{\mu^{\prime}} \in U$, and hence $W^{\mu} \subseteq U$. On the other hand, if $\phi_{\mu \mu}^{1} U=0$ then for all $u \in U$ and $s \in S_{R}(n, r)$, we have

$$
0=\left\langle\left\langle\phi_{\mu \mu}^{1} s u, \phi_{\mu \omega}^{1} T_{w_{\mu}} y_{\mu^{\prime}}\right\rangle\right\rangle=\left\langle\left\langle u, s^{*} \phi_{\mu \omega}^{1} T_{w_{\mu}} y_{\mu^{\prime}}\right\rangle\right\rangle
$$

(because $\phi_{\mu \mu}^{1 *}=\phi_{\mu \mu}^{1}$ and $\phi_{\mu \mu}^{1} \phi_{\mu \omega}^{1}=\phi_{\mu \omega}^{1}$ ), and so $\left\langle\left\langle u, s^{*} z_{\mu}\right\rangle\right\rangle=0$. Thus $U \subseteq W^{\mu \perp}$, as desired.

4.5. Lemma. The generator $z_{\mu}$ of $S^{\mu}$ is anisotropic.

Proof. $z_{\mu}=\phi_{\mu \omega}^{1} T_{w_{\mu}} y_{\mu^{\prime}}$ so

$$
\left\langle\left\langle z_{\mu}, z_{\mu}\right\rangle\right\rangle=\left\langle\phi_{\mu \omega}^{1} T_{w_{\mu}}, \phi_{\mu \omega}^{1} T_{w_{\mu}} y_{\mu^{\prime}}\right\rangle=\left\langle x_{\mu} T_{w_{\mu}}, x_{\mu} T_{w_{\mu}} y_{\mu^{\prime}}\right\rangle_{\mu} .
$$

Now, $x_{\mu} T_{w_{\mu}} y_{\mu^{\prime}}=x_{\mu} T_{w_{\mu}}+$ terms $x_{\mu} T_{d}$, where $d \in \mathscr{D}_{\mu}$ and $d \neq w_{\mu}$ (compare $[2,4.1])$. Therefore, $\left\langle\left\langle z_{\mu}, z_{\mu}\right\rangle\right\rangle=\left\langle x_{\mu} T_{w_{\mu}}, x_{\mu} T_{w_{\mu}}\right\rangle_{\mu}=q^{l\left(w_{\mu}\right)} \neq 0$.

By combining our last two results, we have (compare $[2,4.9]$ )

4.6. Theorem. Suppose that $R$ is a field and $\mu \in \Lambda(n, r)$. Then $W^{\mu} \cap$ $W^{\mu \perp}$ is the unique maximal submodule of $W^{\mu}$ and the quotient module $W^{\mu} /\left(W^{\mu} \cap W^{\mu \perp}\right)$ is an absolutely irreducible self-dual $S_{R}(n, r)$-module.

4.7. Definition. Suppose that $R$ is a field and $\mu \in \Lambda(n, r)$. The irreducible $S_{R}(n, r)$-module $W^{\mu} /\left(W^{\mu} \cap W^{\mu \perp}\right)$ is denoted by $F^{\mu}$.

We have seen that if $\lambda$ and $\mu$ are associated compositions then $W^{\lambda}=W^{\mu}$, so in this case $F^{\lambda}=F^{\mu}$. Thus, $q$-Weyl modules and the irreducible modules $F^{\mu}$ are indexed by partitions of $r$.

The next few results are essentially results from [2], but we include the proofs for completeness.

4.8. Lemma (compare $[2,4.5])$. Suppose that $\lambda, \mu \in \Lambda(n, r)$, and

$$
\theta \in \operatorname{Hom}_{S_{R}(n, r)}\left(L^{\mu^{\prime}}, L^{\lambda^{\prime}}\right) .
$$

We have:

(i) If $\lambda$ q $\mu$ then $\operatorname{ker} \theta \geq W^{\mu}$

(ii) If $\mu \nsubseteq \lambda$ then $\operatorname{Im} \theta \leq W^{\lambda \perp}$. 
Proof. (i) Assume that $\operatorname{ker} \theta \nsucceq W^{\mu}$. Note that $\phi_{\mu \mu}^{1} z_{\mu}=z_{\mu}$. We have $0 \neq$ $z_{\mu} \theta=\left(\phi_{\mu \omega}^{1} T_{w_{\mu}} y_{\mu^{\prime}}\right) \theta=\phi_{\mu \mu}^{1}\left(\phi_{\mu \omega}^{1} T_{w_{\mu}} y_{\mu^{\prime}}\right) \theta \in \phi_{\mu \mu}^{1} S_{R}(n, r) y_{\lambda^{\prime}}$. Therefore, $\lambda^{\prime \prime} \unrhd \mu$ by Lemma 3.2. Thus, $\lambda \unrhd \mu$.

(ii) Assume that $u \in L^{\mu^{\prime}}$ and $u \theta \notin W^{\lambda \perp}$. Let $s z_{\lambda}$ be an element of $W^{\lambda}$ with $s \in S_{R}(n, r)$, such that $s z_{\lambda}$ is not orthogonal to $u \theta$. Then

$$
0 \neq\left\langle\left\langle s z_{\lambda}, u \theta\right\rangle\right\rangle=\left\langle\left\langle s \phi_{\lambda \omega}^{1} T_{w_{i^{\prime}}} y_{\lambda^{\prime}}, u \theta\right\rangle\right\rangle=\left\langle\left\langle\phi_{\lambda \omega}^{1} T_{w_{i}} y_{\lambda^{\prime}}, \phi_{\lambda \lambda^{\prime}}^{1} s^{*}(u \theta)\right\rangle\right\rangle .
$$

Therefore, $0 \neq \phi_{\lambda \lambda}^{1} s^{*}(u \theta)=\left(\phi_{\lambda \lambda}^{1} s^{*} u\right) \theta$, so $0 \neq \phi_{\lambda \lambda}^{1} s^{*} u \in \phi_{\lambda \lambda}^{1} S_{R}(n, r) y_{\mu^{\prime}}$. Therefore, $\mu^{\prime \prime} \unrhd \lambda$ by Lemma 3.2. Thus, $\mu \unrhd \lambda$.

4.9. Lemma (compare [2, 4.11]). Suppose that $\lambda, \mu \in \Lambda(n, r)$. Let $U$ be an $S_{R}(n, r)$-submodule of $L^{\mu^{\prime}}$ and let $\theta$ be an $S_{R}(n, r)$-homomorphism from $W^{\lambda}$ into $L^{\mu^{\prime}} / U$. If $\theta$ is nonzero then $\mu \unrhd \lambda$. If $\lambda^{\prime}=\mu^{\prime}$ then $\operatorname{Im} \theta \leq\left(W^{\lambda}+U\right) / U$.

Proof. Assume that $\theta$ is nonzero. Since $z_{\lambda}$ generates $W^{\lambda}$, we find $s \in S_{R}(n, r)$ such that $z_{\lambda} \theta=s y_{\mu^{\prime}}+U$ and $s y_{\mu^{\prime}} \notin U$. Therefore, $z_{\lambda} \theta=\phi_{\lambda \lambda}^{1} z_{\lambda} \theta=\phi_{\lambda \lambda}^{1} s y_{\mu^{\prime}}+$ $U$. In particular, $0 \neq \phi_{\lambda \lambda}^{1} s y_{\mu^{\prime}} \in \phi_{\lambda \lambda}^{1} S_{R}(n, r) y_{\mu^{\prime}}$. By Lemma 3.2, $\mu^{\prime \prime} \unrhd \lambda$, so $\mu \unrhd \lambda$.

If $\lambda^{\prime}=\mu^{\prime}$ then $z_{\lambda} \theta=\phi_{\lambda \lambda}^{1} s y_{\lambda^{\prime}}+U=c z_{\lambda}+U$ for some $c \in R$, by Lemma 3.2. Therefore, $\operatorname{Im} \theta \leq\left(W^{\lambda}+U\right) / U$.

Applying the results of the last two lemmas, we have

4.10. Corollary (compare [2, 4.12]). Suppose that $R$ is a field and $\lambda, \mu \in$ $\Lambda(n, r)$. We have:

(i) If $F^{\lambda}$ occurs as a composition factor of $L^{\mu^{\prime}}$ then $\mu \unrhd \lambda$.

(ii) If $F^{\lambda}$ occurs as a composition factor of $L^{\mu^{\prime}} / W^{\mu}$ then $\mu \triangleright \lambda$.

4.11. Corollary (compare [2, 4.13]). Suppose that $R$ is a field and $\lambda, \mu \in$ $\Lambda(n, r)$. We have $F^{\lambda} \cong F^{\mu}$ if and only if $\lambda$ and $\mu$ are associated compositions. Thus, $\left\{F^{\lambda} \mid \lambda \vdash r\right\}$ is a set of nonisomorphic absolutely irreducible self-dual $S_{R}(n, r)$-modules.

One of our goals is to prove that every irreducible $S_{R}(n, r)$-module (when $R$ is a field) is isomorphic to some $F^{\lambda}$. That is, $\left\{F^{\lambda} \mid \lambda \vdash r\right\}$ is complete.

If $R$ is a field and $1+q+q^{2}+\cdots+q^{i-1} \neq 0$ for all $i$ with $1 \leq i \leq r$ then the Hecke algebra is semisimple and has precisely as many pairwise nonisomorphic irreducible modules as there are partitions of $r[2,4.3]$. In these circumstances, it follows that the $q$-Schur algebra is also semisimple with as many irreducibles as there are partitions of $r$. By Theorem 4.6, $F^{\lambda}=W^{\lambda}$ in this case, so $\left\{W^{\lambda} \mid \lambda \vdash r\right\}$ is a complete set of irreducible $S_{R}(n, r)$-modules.

We now give two more corollaries of Lemmas 4.8 and 4.9 in the case where $R$ is a field, but without assuming that the $q$-Schur algebra is semisimple. 
4.12. Corollary (compare [2, 4.14]). Suppose that $R$ is a field and $\mu \in \Lambda(n, r)$. Then the multiplicity of $F^{\mu}$ as a composition factor of $L^{\mu^{\prime}}$ and of $W^{\mu}$ is one. Proof. By Corollary 4.10, $F^{\mu}$ is not a composition factor of $L^{\mu^{\prime}} / W^{\mu}$. Hence $F^{\mu}$ is not a composition factor of $\left(L^{\mu^{\prime}} / W^{\mu}\right)^{*}$ which is isomorphic to $W^{\mu \perp}$. In particular, $F^{\mu}$ is not a composition factor of $W^{\mu} \cap W^{\mu \perp}$. Since $F^{\mu}=$ $W^{\mu} /\left(W^{\mu} \cap W^{\mu \perp}\right)$, the corollary now follows by considering the series $W^{\mu} \cap$ $W^{\mu \perp} \leq W^{\mu} \leq L^{\mu^{\prime}}$.

By combining Corollaries 4.10 and 4.12, we have

4.13. Corollary. Suppose that $R$ is a field. For $\lambda$ and $\mu$ partitions of $r$, let $d_{\lambda \mu}$ be the multiplicity of $F^{\mu}$ as a composition factor of $W^{\lambda}$. If the partitions of $r$ are ordered lexiographically then the matrix $\left(d_{\lambda \mu}\right)$ is upper unitriangular.

We conclude this section with a description of the unique maximal submodule of the $q$-Weyl module in terms of $q$-weight spaces (compare [5, 5.4b]).

4.14. Lemma. Suppose that $R$ is a field and that $\mu \in \Lambda(n, r)$. Let

$$
r_{\mu}\left(W^{\mu}\right)=\left\{v \in W^{\mu} \mid \phi_{\mu \mu}^{1} v=0\right\} .
$$

Then $r_{\mu}\left(W^{\mu}\right)$ is a proper subspace of $W^{\mu}$, and for every proper $S_{R}(n, r)$ submodule $U$ of $W^{\mu}$, we have $U \subseteq r_{\mu}\left(W^{\mu}\right)$.

Proof. Since $\phi_{\mu \mu}^{1} z_{\mu}=z_{\mu} \neq 0$, we see that $r_{\mu}\left(W^{\mu}\right) \neq W^{\mu}$. Suppose that $U$ is an $S_{R}(n, r)$-submodule of $W^{\mu}$, but $U \nsubseteq r_{\mu}\left(W^{\mu}\right)$. Then for some $u \in U$ we have $u \notin r_{\mu}\left(W^{\mu}\right)$, so $\phi_{\mu \mu}^{1} u \neq 0$. By Lemma 3.2, $\phi_{\mu \mu}^{1} u=c z_{\mu}$ for some nonzero $c \in R$. Therefore, $z_{\mu} \in U$ and $U=W^{\mu}$.

From Lemma 4.14, we see that the sum of all proper submodules of $W^{\mu}$ is contained in $r_{\mu}\left(W^{\mu}\right)$ and so is a proper submodule of $W^{\mu}$. This provides another proof that $W^{\mu}$ has a unique maximal submodule.

\section{THE LeFT MOdULE $L^{\mu}$ FOR THE $q$-SCHUR Algebra}

In the theory of $q$-Specht modules $S^{\mu}$, it is helpful to work with $M^{\mu}$ which contains $S^{\mu}$. Similarly when dealing with $q$-Weyl modules $W^{\mu}$, the module $L^{\mu^{\prime}}$ which contains $W^{\mu}$ is useful. We shall construct a basis for $L^{\mu}$ in this section. First, we need a combinatorial definition.

5.1. Definition. Suppose that $\lambda$ and $\mu$ are compositions of $r$. Let

$$
\mathscr{C}_{\lambda \mu}=\left\{d \in \mathscr{D}_{\lambda \mu} \mid \mathfrak{S}_{\lambda} \cap d \mathfrak{S}_{\mu} d^{-1}=(1)\right\}
$$

Note that if $d \in \mathscr{C}_{\lambda \mu}$ then every element $w$ of $\mathfrak{S}_{\lambda} d \mathfrak{S}_{\mu}$ has a unique expression in the form $w=u d v$ with $u \in \mathfrak{S}_{\lambda}$ and $v \in \mathfrak{S}_{\mu}$. Also, $l(w)=$ $l(u)+l(d)+l(v)$. 
5.2. Theorem. Suppose that $\mu \in \Lambda(n, r)$. Then $L^{\mu}$ is free as an $R$-module, with basis

$$
\left\{\phi_{\lambda \omega}^{1} T_{d} y_{\mu} \mid \lambda \in \Lambda(n, r) \text { and } d \in \mathscr{C}_{\lambda \mu}\right\} \text {. }
$$

Proof. Fix $\lambda \in \Lambda(n, r)$. If $d \in \mathscr{C}_{\lambda \mu}$ then $x_{\lambda} T_{d} y_{\mu}=\sum_{u \in \mathfrak{S}_{\lambda}} \sum_{v \in \mathfrak{S}_{\mu}}(-q)^{-l(v)} T_{u d v}$ $\neq 0$. Hence $\left\{x_{\lambda} T_{d} y_{\mu} \mid d \in \mathscr{C}_{\lambda \mu}\right\}$ is a linearly independent subset of $\mathscr{H}$. Thus $\left\{\phi_{\lambda \omega}^{1} T_{d} y_{\mu} \mid d \in \mathscr{C}_{\lambda \mu}\right\}$ is a linearly independent subset of $L^{\mu} \cap M^{\lambda}$.

Now, $L^{\mu} \cap M^{\lambda}=\phi_{\lambda \lambda}^{1} E_{R}(n, r) y_{\mu}$ and $\left\{\phi_{\lambda \omega}^{1} T_{d} \mid d \in \mathscr{D}_{\lambda}\right\}$ is a basis of $M^{\lambda}$. Therefore, the elements $\phi_{\lambda \omega}^{1} T_{d} y_{\mu}$ with $d \in \mathscr{D}_{\lambda}$ generate $L^{\mu} \cap M^{\lambda}$. For $d \in \mathscr{D}_{\lambda}$, let $\bar{d}$ denote the distinguished double coset representative of $\mathfrak{S}_{\lambda} d \mathfrak{S}_{\mu}$. Then $d=\bar{d} v$ for some $v \in \mathfrak{S}_{\mu}$ such that $l(d)=l(\bar{d})+l(v)$. Hence, we have

$$
\phi_{\lambda \omega}^{1} T_{d} y_{\mu}=\phi_{\lambda \omega}^{1} T_{\bar{d}} T_{v} y_{\mu}=(-1)^{l(v)} \phi_{\lambda \omega}^{1} T_{\bar{d}} y_{\mu}
$$

Thus, $\left\{\phi_{\lambda \omega}^{1} T_{d} y_{\mu} \mid d \in \mathscr{D}_{\lambda \mu}\right\}$ generates $L^{\mu} \cap M^{\lambda}$.

Finally, suppose that $d \in \mathscr{D}_{\lambda \mu} \backslash \mathscr{C}_{\lambda \mu}$. Then $\mathfrak{S}_{\lambda} \cap d \mathfrak{S}_{\mu} d^{-1}$ contains a basic transposition, a say, and $d^{-1} a d$ is a basic transposition, $d^{-1} a d=b$ say, and $T_{a} T_{d}=T_{d} T_{b}$ (compare $[2,1.6]$ ). Since $b \in \mathfrak{S}_{\mu}$, we may write $y_{\mu}$ as $\left(1-q^{-1} T_{b}\right) \tilde{y}_{\mu}$ for some $\tilde{y}_{\mu} \in S_{R}(n, r)$. Then $\phi_{\lambda \omega}^{1} T_{d} y_{\mu}=\phi_{\lambda \omega}^{1} T_{d}\left(1-q^{-1} T_{b}\right) \tilde{y}_{\mu}=$ $\phi_{\lambda \omega}^{1}\left(1-q^{-1} T_{a}\right) T_{d} \tilde{y}_{\mu}$. But $\phi_{\lambda \omega}^{1} T_{a}=q \phi_{\lambda \omega}^{1}$ since $a \in \mathfrak{S}_{\lambda}$, by result $(2.11)$. Therefore, $\phi_{\lambda \omega}^{1} T_{d} y_{\mu}=0$ if $d \in \mathscr{D}_{\lambda \mu} \backslash \mathscr{C}_{\lambda \mu}$.

We have now proved that $\left\{\phi_{\lambda \omega}^{1} T_{d} y_{\mu} \mid d \in \mathscr{C}_{\lambda \mu}\right\}$ generates $L^{\mu} \cap M^{\lambda}$, and since $L^{\mu}=\bigoplus_{\lambda \in \Lambda(n, r)}\left(L^{\mu} \cap M^{\lambda}\right)$ by Theorem 2.14 , the proof of the theorem is now complete.

\section{SETS OF HOMOMORPHISMS}

It was observed by Carter and Lusztig [1] that tensor space contains copies of various important spaces of homomorphisms. We investigate analogues of their results for $q$-tensor space in this section.

If $X$ is a subset of the $q$-Schur algebra, then the left annihilator $l(X)$ is the left ideal $\left\{s \in S_{R}(n, r) \mid s x=0\right.$ for all $\left.x \in X\right\}$ of $S_{R}(n, r)$, and the right annihilator $r(X)$ is defined in a similar way. If $X$ is a set with just one element, $X=\{s\}$, say, then we use the abbreviations $r(X)=r(s)$ and $l(X)=l(s)$.

6.1. Lemma. Suppose that $\mu \in \Lambda(n, r)$.

(i) If $s \in \phi_{\mu \mu}^{1} S_{R}(n, r)$ then $r l(s) \leq \phi_{\mu \mu}^{1} S_{R}(n, r)$.

(ii) If $s \in S_{R}(n, r) \phi_{\mu \mu}^{1}$, then $\operatorname{lr}(s) \leq S_{R}(n, r) \phi_{\mu \mu}^{1}$.

Proof. Suppose that $s \in \phi_{\mu \mu}^{1} S_{R}(n, r)$ and that $v=r l(s)$. We have $v=$ $\sum_{\lambda \in \Lambda(n, r)} \phi_{\lambda \lambda}^{1} v$ by result (2.4). But if $\lambda \neq \mu$ then $\phi_{\lambda \lambda}^{1} s=0$ so $\phi_{\lambda \lambda}^{1} v=0$. 
Therefore, $v=\phi_{\mu \mu}^{1} v \in \phi_{\mu \mu}^{1} S_{R}(n, r)$. This completes the proof of part (i) of the lemma, and the proof of part (ii) is similar.

6.2. Lemma. Suppose that $s$ belongs to q-tensor space. Then

$$
\operatorname{lr}(s)=\left\{v \in E_{R}(n, r) \mid \text { if } h \in \mathscr{H} \text { and } s h=0 \text {, then } v h=0\right\} .
$$

Proof. If $v \in \operatorname{lr}(s)$, then $v \in E_{R}(n, r)$ by Lemma 6.1(ii), and $v h=0$ for all $h \in \mathscr{H}$ such that $s h=0$.

Conversely, suppose that $v \in E_{R}(n, r)$ and $v h=0$ for all $h \in \mathscr{H}$ such that $s h=0$. Let $u \in r(s)$. We must prove that $v u=0$. We have $0=s u=s \phi_{\omega \omega}^{1} u$, so $s \phi_{\omega \omega}^{1} u \phi_{\lambda \omega}^{1}=0$ for all $\lambda \in \Lambda(n, r)$. But $\phi_{\omega \omega}^{1} u \phi_{\lambda \omega}^{1} \in \mathscr{H}$, so $v \phi_{\omega \omega}^{1} u \phi_{\lambda \omega}^{1}=0$. Therefore, $v \phi_{\omega \omega}^{1} u \phi_{\lambda \lambda}^{1}=0$, by Lemma 2.5. By summing over $\lambda \in \Lambda(n, r)$, we obtain $v \phi_{\omega \omega}^{1} u=0$. But $v \in E_{R}(n, r)$ so $v \phi_{\omega \omega}^{1}=v$. We have now proved that $v u=0$, as desired.

6.3. Lemma. Suppose that $s$ belongs to q-tensor space.

(i) If $U$ is an $\mathscr{H}$-submodule of q-tensor space, then the $R$-modules $\operatorname{Hom}_{\mathscr{H}}(s \mathscr{H}, U)$ and $\operatorname{lr}(s) \cap U$ are canonically isomorphic.

(ii) If $U$ is an $S_{R}(n, r)$-submodule of q-tensor space, then the $R$-modules $\operatorname{Hom}_{S_{R}(n, r)}\left(S_{R}(n, r) s, U\right)$ and $r l(s) \cap U$ are canonically isomorphic.

Proof. (i) Suppose that $U$ is an $\mathscr{H}$-submodule of $q$-tensor space. If $\theta \in$ $\operatorname{Hom}_{\mathscr{H}}(s \mathscr{H}, U)$ then $\theta s \in \operatorname{lr}(s) \cap U$, by Lemma 6.2. On the other hand, if $u \in \operatorname{lr}(s) \cap U$, then $s h \mapsto u h(h \in \mathscr{H})$ gives an element of $\operatorname{Hom}_{\mathscr{H}}(s \mathscr{H}, U)$. Hence $\theta \mapsto \theta s$ is the required isomorphism.

(ii) The proof of part (ii) is similar to the proof of part (i), but is simpler, since Lemma 6.2 is not needed.

6.4. Theorem. Suppose that $\mu \in \Lambda(n, r)$. Then

(i) $M^{\mu}=\operatorname{rl}\left(\phi_{\mu \omega}^{1}\right) \cap E_{R}(n, r)$ and

(ii) $S^{\mu}=r l\left(z_{\mu}\right) \cap E_{R}(n, r)$.

Proof. (i) Recall that $M^{\mu}=\phi_{\mu \omega}^{1} \mathscr{H}=\phi_{\mu \mu}^{1} S_{R}(n, r) \phi_{\omega \omega}^{1}$. Clearly, $M^{\mu} \leq r l\left(\phi_{\mu \omega}^{1}\right)$ $\cap E_{R}(n, r)$. On the other hand, by Lemma 6.1(i),

$$
\begin{aligned}
r l\left(\phi_{\mu \omega}^{1}\right) \cap E_{R}(n, r) & \leq\left(\phi_{\mu \mu}^{1} S_{R}(n, r)\right) \cap\left(S_{R}(n, r) \phi_{\omega \omega}^{1}\right) \\
& =\phi_{\mu \mu}^{1} S_{R}(n, r) \phi_{\omega \omega}^{1}=M^{\mu},
\end{aligned}
$$

and this completes the proof of result (i).

(ii) Again, one inclusion, namely $S^{\mu}=z_{\mu} \mathscr{H} \leq r l\left(z_{\mu}\right) \cap E_{R}(n, r)$, is clear. Suppose, therefore, that $v \in \operatorname{rl}\left(z_{\mu}\right) \cap E_{R}(n, r)$. Then $v \in M^{\mu}$ by Lemma 6.1(i). If $\lambda \in \Lambda(n, r)$ with $\mu \notin \lambda$ and $d \in \mathscr{D}_{\lambda \mu}$ then $\phi_{\lambda \mu}^{d} z_{\mu} \in \phi_{\lambda \lambda}^{1} S_{R}(n, r) y_{\mu^{\prime}}=(0)$, by Lemma 3.2. Therefore, $\phi_{\lambda \mu}^{d} v=0$. By applying Corollary 3.7, we deduce that $v \in S^{\mu}$, as desired.

We remark that it is not hard to show that $r l\left(\phi_{\mu \omega}^{1}\right)=\phi_{\mu \mu}^{1} S_{R}(n, r)$. 
Next, notice that if $U$ is an $S_{R}(n, r)$-submodule of $q$-tensor space, then we obtain from each element $h$ of $\mathscr{H}$ an $S_{R}(n, r)$-homomorphism from $U$ into $q$-tensor space by $u \mapsto u h \quad(u \in U)$. Similarly, if $U$ is an $\mathscr{U}$-submodule of $q$ tensor space, then each element $s$ of $S_{R}(n, r)$ gives us an $\mathscr{H}$-homomorphism $u \mapsto s u(u \in U)$ from $U$ into $q$-tensor space. It is of interest to know whether every homomorphism is given in this simple way.

6.5. Lemma. Suppose that $\mu \in \Lambda(n, r)$ and that $U$ is an $\mathscr{H}$-submodule of q-tensor space. Then

(i) Every element of $\operatorname{Hom}_{\mathscr{H}}\left(M^{\mu}, E_{R}(n, r)\right)$ is given by left multiplication by some element of $S_{R}(n, r)$.

(ii) The R-modules $\operatorname{Hom}_{\mathscr{H}}\left(M^{\mu}, U\right)$ and $S_{R}(n, r) \phi_{\mu \omega}^{1} \cap U$ are canonically isomorphic.

Proof. Suppose that $\lambda \in \Lambda(n, r)$ and $\theta \in \operatorname{Hom}_{\mathscr{H}}\left(M^{\mu}, M^{\lambda}\right)$. Then $\theta\left(\phi_{\mu \omega}^{1}\right)=$ $\phi_{\lambda \omega}^{1} h_{\theta}$ for some $h_{\theta} \in \mathscr{H}$. Since $\phi_{\mu \omega}^{1} \mathscr{H}$ and $x_{\mu} \mathscr{H}$ are canonically isomorphic, the map $x_{\mu} h \mapsto x_{\lambda} h_{\theta} h(h \in \mathscr{H})$ is an element of $\operatorname{Hom}_{\mathscr{H}}\left(x_{\mu} \mathscr{H}, x_{\lambda} \mathscr{H}\right)$. Therefore, $x_{\lambda} h_{\theta}=s_{\theta}\left(x_{\mu}\right)$ for some $s_{\theta} \in S_{R}(n, r)$. It is easy to check that $\phi_{\lambda \omega}^{1} h_{\theta}=s_{\theta} \phi_{\mu \omega}^{1}$. Therefore, $\theta$ is obtained by multiplying on the left side by $s_{\theta}$. Since $\operatorname{Hom}_{\mathscr{H}}\left(M^{\mu}, E_{R}(n, r)\right)=\bigoplus_{\lambda \in \Lambda(n, r)} \operatorname{Hom}_{\mathscr{H}}\left(M^{\mu}, M^{\lambda}\right)$, every element of $\operatorname{Hom}_{\mathscr{H}}\left(M^{\mu}, E_{R}(n, r)\right)$ is given by left multiplication by some element of $S_{R}(n, r)$.

The isomorphism between $\operatorname{Hom}_{\mathscr{H}}\left(M^{\mu}, U\right)$ and $S_{R}(n, r) \phi_{\mu \omega}^{1} \cap U$ is given by $\theta \mapsto \theta \phi_{\mu \omega}^{1}$.

6.6. Theorem (the double centralizer property).

(i) $\operatorname{End}_{S_{R}(n, r)}\left(E_{R}(n, r)\right)=\mathscr{H}$.

(ii) $\operatorname{End}_{\mathscr{H}}\left(E_{R}(n, r)\right)=S_{R}(n, r)$.

Proof. The first result holds since

$$
E_{R}(n, r)=S_{R}(n, r) \phi_{\omega \omega}^{1} \text { and } \mathscr{H}=\phi_{\omega \omega}^{1} S_{R}(n, r) \phi_{\omega \omega}^{1}
$$

and $\phi_{\omega \omega}^{1}$ is an idempotent. The second result follows from Lemma 6.5(i) and the fact that $\operatorname{End}_{\mathscr{H}}\left(E_{R}(n, r)\right)=\bigoplus_{\lambda \in \Lambda(n, r)} \operatorname{Hom}_{\mathscr{H}}\left(M^{\lambda}, E_{R}(n, r)\right)$.

6.7. Lemma. Suppose that $\lambda, \mu \in \Lambda(n, r)$ and that $U$ is an $S_{R}(n, r)$-submodule of q-tensor space. Then

(i) Every element of $\operatorname{Hom}_{S_{R}(n, r)}\left(L^{\mu}, E_{R}(n, r)\right)$ is given by right multiplication by some element of $\mathscr{H}$.

(ii) The $R$-modules $\operatorname{Hom}_{S_{R}(n, r)}\left(L^{\mu}, U\right)$ and $y_{\mu} \mathscr{H} \cap U$ are canonically isomorphic.

(iii) The R-modules $\operatorname{Hom}_{S_{R}(n, r)}\left(L^{\mu}, L^{\lambda}\right)$ and $\operatorname{Hom}_{\mathscr{H}}\left(\mathscr{H} y_{\mu}, \mathscr{H} y_{\lambda}\right)$ are canonically isomorphic. 
Proof. For $\theta \in \operatorname{Hom}_{S_{R}(n, r)}\left(L^{\mu}, L^{\lambda}\right)$, let $\bar{\theta}$ denote the restriction of $\theta$ to $\mathscr{H} y_{\mu}$. We have $y_{\mu} \theta=\phi_{\omega \omega}^{1} y_{\mu} \theta \in \phi_{\omega \omega}^{1} E_{R}(n, r) y_{\lambda}=\mathscr{H} y_{\lambda}$, so $\bar{\theta} \in \operatorname{Hom}_{\mathscr{H}}\left(\mathscr{H} y_{\mu}, \mathscr{H} y_{\lambda}\right)$. But every element of $\operatorname{Hom}_{\mathscr{H}}\left(\mathscr{H} y_{\mu}, \mathscr{H} y_{\lambda}\right)$ is given by right multiplication by an element of $\mathscr{H}[3,3.7]$. Moreover, $\bar{\theta}$ corresponds to right multiplication by $h \in \mathscr{H}$ if and only if $\left(s y_{\mu}\right) \theta=s\left(y_{\mu} \theta\right)=s y_{\mu} h$ for all $s \in S_{R}(n, r)$, that is, if and only if $\theta$ corresponds to right multiplication by $h$. In particular, $\bar{\theta}=0$ if and only if $\theta=0$.

Part (i) of the lemma is obtained by noting that $E_{R}(n, r)=L^{\lambda}$, where $\lambda=\left(1^{r}\right)$. The isomorphism in part (ii) of the lemma is given by $\theta \mapsto y_{\mu} \theta$. Finally, $\theta \rightarrow \bar{\theta}$ gives the isomorphism of part (iii) of the lemma.

6.8. Lemma. Suppose that $\mu \in \Lambda(n, r)$ and that $U$ is an $S_{R}(n, r)$-submodule of q-tensor space. Then

(i) Every element of $\operatorname{Hom}_{S_{R}(n, r)}\left(W^{\mu}, E_{R}(n, r)\right)$ is given by right multiplication by some element of $\mathscr{H}$.

(ii) The R-modules $\operatorname{Hom}_{S_{R}(n, r)}\left(W^{\mu}, U\right)$ and $S^{\mu} \cap U$ are canonically isomorphic.

Proof. Suppose that $\theta \in \operatorname{Hom}_{S_{R}(n, r)}\left(W^{\mu}, U\right)$. Then $z_{\mu} \theta \in r l\left(z_{\mu}\right) \cap U$. Therefore $z_{\mu} \theta=z_{\mu} h$ for some $h \in \mathscr{H}$ by Theorem 6.4(ii). Part (i) of the lemma follows immediately. The isomorphism in part (ii) of the lemma is given by $\theta \mapsto z_{\mu} \theta$.

Lemmas 6.7 and 6.8 show that every homomorphism from $L^{\mu}$ or $W^{\mu}$ into $q$-tensor space is given by right multiplication, and Lemma 6.5 shows that every homomorphism from $M^{\mu}$ into $q$-tensor space is given by left multiplication. The situation for $q$-Specht modules $S^{\mu}$ is more complicated.

6.9. Lemma. Suppose that $\lambda, \mu \in \Lambda(n, r)$. Let $\mathscr{E}$ denote the set of $\mathscr{H}$ homomorphisms from $S^{\mu}$ into $M^{\lambda}$ which can be extended to an element of $\operatorname{Hom}_{\mathscr{H}}\left(M^{\mu}, M^{\lambda}\right)$. Then $\operatorname{Hom}_{\mathscr{H}}\left(S^{\mu}, M^{\lambda}\right)$ is canonically isomorphic, as an $R$ module, to $\operatorname{lr}\left(z_{\mu}\right) \cap M^{\lambda}$, and the restriction of this isomorphism to $\mathscr{E}$ maps onto $W^{\mu} \cap M^{\lambda}$.

Proof. By Lemma 6.3(i), $\theta \mapsto \theta z_{\mu} \quad\left(\theta \in \operatorname{Hom}_{\mathscr{H}}\left(S^{\mu}, M^{\lambda}\right)\right)$ gives a canonical isomorphism between $\operatorname{Hom}_{\mathscr{H}}\left(S^{\mu}, M^{\lambda}\right)$ and $\operatorname{lr}\left(z_{\mu}\right) \cap M^{\lambda}$. If $\theta \in \mathscr{E}$ then $\theta$ is given by left multiplication by some element of $S_{R}(n, r)$, by Lemma 6.5, so $\theta z_{\mu} \in W^{\mu}$. On the other hand, if $s z_{\mu} \in W^{\mu} \cap M^{\lambda}$ then $\theta: z_{\mu} h \mapsto s z_{\mu} h$ $(h \in \mathscr{H})$ gives an element of $\mathscr{E}$, and this remark completes the proof of the lemma.

Of course, we have $W^{\mu} \leq \operatorname{lr}\left(z_{\mu}\right)$, but we shall see later (Example (6.14)) that this inclusion can be strict, and in such cases, not every element of $\operatorname{Hom}_{\mathscr{Z}}\left(S^{\mu}, M^{\lambda}\right)$ can be extended to an element of $\operatorname{Hom}_{\mathscr{H}}\left(M^{\mu}, M^{\lambda}\right)$. We investigate the module $\operatorname{lr}\left(z_{\mu}\right)$ by first showing that $\operatorname{lr}\left(y_{\mu}\right)$ is often equal to $L^{\mu}$ : 
6.10. Theorem. Suppose that $\mu \in \Lambda(n, r)$. Assume that $q+1 \neq 0$. Then $L^{\mu}=\operatorname{lr}\left(y_{\mu}\right)$.

Proof. Since $L^{\mu}=S_{R}(n, r) y_{\mu}$, it is clear that $L^{\mu} \leq \operatorname{lr}\left(y_{\mu}\right)$.

Suppose that $v \in \operatorname{lr}\left(y_{\mu}\right)$. Then $v$ belongs to $q$-tensor space by Lemma 6.1(ii). For each $\lambda \in \Lambda(n, r)$, let $v_{\lambda}=\phi_{\lambda \lambda}^{1} v$. Then $v=\sum_{\lambda \in \Lambda(n, r)} v_{\lambda}$, so it is sufficient to prove that $v_{\lambda} \in L^{\mu}$. We have $v_{\lambda}=\sum_{d \in \mathscr{D}_{\lambda}} c_{d} \phi_{\lambda \omega}^{d}$ for certain coefficients $c_{d} \in R$.

Next, we divide up $\mathscr{D}_{\lambda}$ in the following way (compare $[2,1.6]$ ). If $d \in$ $\mathscr{D}_{\lambda \mu}$, then define $\nu(d)$ to be the composition of $r$ which corresponds to $\mathfrak{S}_{\lambda} \cap$ $d \mathfrak{S}_{\mu} d^{-1}$. We have

$$
\mathscr{D}_{\lambda}=\bigcup_{d \in \mathscr{D}_{i \mu}} d\left(\mathscr{D}_{\nu(d)} \cap \mathfrak{S}_{\mu}\right)
$$

a disjoint union, so

$$
v_{\lambda}=\sum_{d \in \mathscr{D}_{i, \mu}} \sum_{u \in \mathscr{D}_{\nu(d)} \cap \mathfrak{S}_{\mu}} c_{d u} \phi_{\lambda \omega}^{d u}
$$

We claim tht $c_{d u}=(-q)^{-l(u)} c_{d}$ if $d \in \mathscr{D}_{\lambda \mu}$ and $u \in \mathscr{D}_{\nu(d)} \cap \mathfrak{S}_{\mu}$. We prove this result by induction on $l(u)$. Since the result is immediate if $l(u)=0$, we may assume that $u=w a$ where $a$ is a basic transposition and $l(u)=l(w)+1$. Then $w \in \mathscr{D}_{\nu(d)} \cap \mathfrak{S}_{\mu}$ and $a \in \mathfrak{S}_{\mu}$. Since $a \in \mathfrak{S}_{\mu}$, we have $y_{\mu}\left(1+T_{a}\right)=0$, and since $v_{\lambda} \in \operatorname{lr}\left(y_{\mu}\right)$, we have $v_{\lambda}\left(1+T_{a}\right)=0$.

Now if $\tilde{u} \in \mathscr{D}_{\nu(d)} \cap \mathfrak{S}_{\mu}$ and $\tilde{u}$ is distinct from $u$ and $w$, then diu is distinct from $d u$ and $d w$. Therefore, when we postmultiply $v_{\lambda}$ by $T_{a}$, and apply result (2.11), we get $c_{d w} \phi_{\lambda \omega}^{d u}+c_{d u}\left(q \phi_{\lambda \omega}^{d w}+(q-1) \phi_{\lambda \omega}^{d u}\right)+($ a linear combination of terms which do not involve $\phi_{\lambda \omega}^{d u}$ or $\left.\phi_{\lambda \omega}^{d w}\right)$. Therefore, $v_{\lambda}\left(1+T_{a}\right)=\left(q c_{d u}+c_{d w}\right)\left(\phi_{\lambda \omega}^{d u}+\phi_{\lambda \omega}^{d w}\right)+$ other terms. Since $v_{\lambda}\left(1+T_{a}\right)=0$, we obtain $c_{d u}=(-q)^{-1} c_{d w}=(-q)^{-l(u)} c_{d}$, by induction.

Now suppose that $d \in \mathscr{D}_{\lambda \mu} \mid \mathscr{C}_{\lambda \mu}$. We shall prove that $c_{d}=0$. Since $d \notin$ $\mathscr{C}_{\lambda \mu}$, there exists a basic transposition, a say, in $\mathfrak{S}_{\lambda} \cap d \mathfrak{S}_{\mu} d^{-1}$, and $d^{-1} a d$ is a basic transposition, $d^{-1} a d=b$ say. Now, $v_{\lambda}\left(1+T_{b}\right)=0$ since $b \in \mathfrak{S}_{\mu}$. Also, $\phi_{\lambda \omega}^{d} T_{b}=q \phi_{\lambda \omega}^{d}$ since $l(d b)=l(d)+1$ and $d b=a d \notin \mathscr{D}_{\lambda}$ (see result (2.11)). Therefore,

$$
\begin{aligned}
0 & =v_{\lambda}\left(1+T_{b}\right)=\left(c_{d} \phi_{\lambda \omega}^{d}+\text { other terms }\right)\left(1+T_{b}\right) \\
& =(1+q) c_{d} \phi_{\lambda \omega}^{d}+\text { other terms. }
\end{aligned}
$$

Since $1+q \neq 0$, we have $c_{d}=0$.

We now put together the results which we have proved. If $d \in \mathscr{D}_{\lambda \mu} \backslash \mathscr{C}_{\lambda \mu}$ and $u \in \mathscr{D}_{\nu(d)} \cap \mathfrak{S}_{\mu}$ then $c_{d u}=(-q)^{-l(u)} c_{d}=0$. If $d \in \mathscr{C}_{\lambda \mu}$ then $\mathscr{D}_{\nu(d)} \cap \mathfrak{S}_{\mu}=\mathfrak{S}_{\mu}$ 
and $c_{d u}=(-q)^{-l(u)} c_{d}$ for all $u \in \mathfrak{S}_{\mu}$. Therefore,

$$
v_{\lambda}=\sum_{d \in \mathscr{C}_{\lambda, \mu}} \sum_{u \in \mathfrak{S}_{\mu}}(-q)^{-l(u)} c_{d} \phi_{\lambda \omega}^{d u} .
$$

But $\phi_{\lambda \omega}^{d u}=\phi_{\lambda \omega}^{d} T_{u}$. Thus, $v_{\lambda}=\sum_{d \in \mathscr{C}_{\lambda \mu}} c_{d} \phi_{\lambda \omega}^{d} y_{\mu} \in S_{R}(n, r) y_{\mu}$. That is, $v_{\lambda} \in L^{\mu}$, as we wished to show.

Applying Lemma 6.2 to the result of the last theorem, we obtain

6.11. Corollary. Suppose that $\mu \in \Lambda(n, r)$. Assume that $q+1 \neq 0$. Then

$$
L^{\mu}=\left\{v \in E_{R}(n, r) \mid \text { if } h \in \mathscr{H} \text { and } y_{\mu} h=0 \text { then } v h=0\right\} .
$$

We also have

$$
\operatorname{lr}\left(z_{\mu}\right)=\left\{v \in E_{R}(n, r) \mid \text { if } h \in \mathscr{H} \text { and } z_{\mu} h=0 \text { then } v h=0\right\} .
$$

A composition $\mu$ is said to be 2-regular if all its nonzero parts are distinct.

6.13. Theorem. Suppose that $\mu \in \Lambda(n, r)$. If $q+1 \neq 0$ or $\mu$ is 2-regular, then $\operatorname{lr}\left(z_{\mu}\right) \subseteq L^{\mu^{\prime}}$.

Proof. We have $r\left(y_{\mu^{\prime}}\right) \subseteq r\left(z_{\mu}\right)$, so $\operatorname{lr}\left(z_{\mu}\right) \subseteq \operatorname{lr}\left(y_{\mu^{\prime}}\right)$. If $q+1 \neq 0$ then $\operatorname{lr}\left(y_{\mu^{\prime}}\right)=$ $L^{\mu^{\prime}}$ by Theorem 6.10 , so the conclusion of the theorem is correct in this case.

Now suppose that $\mu$ is 2-regular. By [2,4.10], we find $h \in \mathscr{H}$ and $0 \neq c \in R$ such that $x_{\mu} T_{w_{\mu}} y_{\mu^{\prime}} h y_{\mu^{\prime}}=c x_{\mu} T_{w_{\mu}} y_{\mu^{\prime}}$. That is, $z_{\mu}\left(c-h y_{\mu^{\prime}}\right)=0$. Assume that $v \in \operatorname{lr}\left(z_{\mu}\right)$. Then $v\left(c-h y_{\mu^{\prime}}\right)=0$, and so $c v=v h y_{\mu^{\prime}} \in L^{\mu^{\prime}}$. In the case where $R$ is a field, we immediately deduce that $v \in L^{\mu^{\prime}}$, as we want. In general, from Theorem 5.2 we deduce easily that $L^{\mu^{\prime}}$ has an $R$-complement in $E_{R}(n, r)$ and therefore $L^{\mu^{\prime}}=E_{R}(n, r) \cap L_{F}^{\mu^{\prime}}$, where $L_{F}^{\mu^{\prime}}$ denotes $L^{\mu^{\prime}}$ defined over the quotient field $F$ of $R$. Hence $v \in L^{\mu^{\prime}}$ in general.

The following simple example illustrates the need for the special hypotheses in Theorems 6.10 and 6.13 .

6.14. Example. Let $r=2 \leq n$. We have $x_{(2)}=1+T_{(12)}$ and $y_{(2)}=1-q^{-1} T_{(12)}$. It is easy to calculate that $\left\{h \in \mathscr{H} \mid y_{(2)} h=0\right\}=x_{(2)} \mathscr{H}$, and that $\phi_{(2) \omega} x_{(2)}=$ $(q+1) \phi_{(2) \omega}$. Hence if $q+1=0$ then

$$
\phi_{(2) \omega} \in\left\{v \in E_{R}(n, r) \mid \text { if } h \in \mathscr{C} \text { and } y_{(2)} h=0 \text { then } v h=0\right\}=\operatorname{lr}\left(y_{(2)}\right) \text {, }
$$

by Lemma 6.2. But $\phi_{(2) \omega} \notin L^{(2)}$ (for example, because $\phi_{(2) \omega} \in M^{(2)}$, and $L^{(2)} \cap M^{(2)}=0$ since $\mathscr{C}_{(2)(2)}$ is empty-see Theorem 5.2). Therefore, when $q+1=0$, we have that $L^{(2)}$ is a proper subset of $\operatorname{lr}\left(y_{(2)}\right)$. Thus, we cannot omit the hypothesis that $q+1 \neq 0$ from Theorem 6.10. Also, if $\mu=\left(1^{2}\right)$ then $z_{\mu}=y_{(2)}$, so $\operatorname{lr}\left(z_{\mu}\right) \nsubseteq L^{\mu^{\prime}}$, in this case where $q+1=0$ and $\mu$ is not 2-regular. This shows why the hypothesis of Theorem 6.13 is needed. 


\section{KERNEL INTERSECTIONS}

One of our main aims is to prove that the top composition factors of $q$-Weyl modules provide a complete set of irreducible $S_{R}(n, r)$-modules when $R$ is a field. There seems to be no way of seeing directly why the $q$-Schur algebra should have precisely as many irreducible modules as there are partitions of $r$, so our proof is circuitous. The principal idea will be to describe the $q$-Weyl module $W^{\mu}$, which is a submodule of $L^{\mu^{\prime}}$, as the intersection of the kernels of various $S_{R}(n, r)$-homomorphisms which are defined on $L^{\mu^{\prime}}$. In the process of proving this, several other things come out, including a basis of the $q$-Weyl module.

Just as a basis of $\operatorname{Hom}_{\mathscr{H}}\left(x_{\mu} \mathscr{H}, x_{\lambda} \mathscr{H}\right)$ is given by $\left\{\phi_{\lambda \mu}^{d} \mid d \in \mathscr{D}_{\lambda \mu}\right\}$, where

$$
\phi_{\lambda \mu}^{d}\left(x_{\mu} h\right)=\sum_{w \in \mathfrak{S}_{i} d \mathfrak{S}_{\mu}} T_{w} h \quad(h \in \mathscr{H}),
$$

we have a basis $\left\{\tilde{\phi}_{\lambda \mu}^{d} \mid d \in \mathscr{D}_{\lambda \mu}\right\}$ of $\operatorname{Hom}_{\lambda}\left(\mathscr{H} y_{\lambda}, \mathscr{C} y_{\mu}\right)$, where

$$
\left(h y_{\lambda}\right) \tilde{\phi}_{\lambda \mu}^{d}=h \sum_{w \in \mathfrak{G}_{\lambda} d \mathfrak{G}_{\mu}}(-q)^{-l(w)} T_{w} \quad(h \in \mathscr{H}) .
$$

This was shown in $[3,3.7]$, and follows from the fact that $\mathscr{H}$ has an outer automorphism which interchanges the roles of $x_{\lambda}$ and $y_{\lambda}$. We shall use $\tilde{\phi}_{\lambda \mu}^{d}$ also to denote the corresponding element of $\operatorname{Hom}_{S_{R}(n, r)}\left(L^{\lambda}, L^{\mu}\right)$ (see Lemma 6.7(iii)).

For the remainder of this section, we shall assume that $\mu$ is a partition of $r$. This assumption involves little loss when studying $q$-Weyl modules, in view of Lemma 3.9.

By Lemma 4.8, we have the following:

(7.1) $W^{\mu^{\prime}} \leq L^{\mu}$, and if $\phi \in \operatorname{Hom}_{S_{R}(n, r)}\left(L^{\mu}, L^{\lambda}\right)$ and $\mu q \lambda$ then $W^{\mu^{\prime}} \phi=0$.

We wish to strengthen this result to give a Kernel Intersection Theorem for $q$-Weyl modules (compare result (3.4) and Theorem 3.6).

7.2. Definition (compare Definition 3.5). Fix $\mu=\left(\mu_{1}, \mu_{2}, \ldots, \mu_{n}\right)$ to be a partition of $r$. If $1 \leq i \leq n-1$ and $0 \leq j \leq \mu_{i+1}$ then let $\tilde{\psi}_{i j}=\tilde{\phi}_{\mu \lambda}^{1} \in$ $\operatorname{Hom}_{S_{R}(n, r)}\left(L^{\mu}, L^{\lambda}\right)$, where $\lambda=\left(\lambda_{1}, \lambda_{2}, \ldots, \lambda_{n}\right)$ is the composition of $r$ for which $\lambda_{i}=\mu_{i}+\mu_{i+1}-j, \lambda_{i+1}=j$, and $\lambda_{a}=\mu_{a}$ if $1 \leq a \leq n$ and $a \neq i$ or $i+1$.

7.3. Definitions. Suppose that $\mu \vdash r$.

(i) Let $K^{\mu}=\bigcap_{i=2}^{k} \bigcap_{j=0}^{\mu_{i}-1} \operatorname{ker} \tilde{\psi}_{i-1, j}$, where $k$ is equal to the number of nonzero parts of $\mu$.

(ii) If $\lambda \in \Lambda(n, r)$, then let $\mathfrak{X}_{\lambda}$ denote the set of $S_{R}(n, r)$-homomorphisms

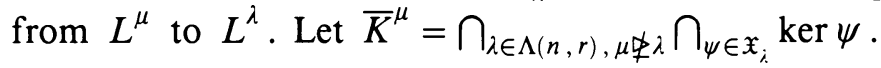

Note that $K^{\mu} \geq \bar{K}^{\mu} \geq W^{\mu^{\prime}}$. In the end, we shall prove that $K^{\mu}=W^{\mu^{\prime}}$. 
7.4. Lemma. We have $K^{\mu}=\bar{K}^{\mu}$.

Proof. Suppose that $v \in K^{\mu}$ and that $\mu \notin \lambda$ and $\theta \in \operatorname{Hom}_{S_{R}(n, r)}\left(L^{\mu}, L^{\lambda}\right)$. We shall be home if we can prove that $v \theta=0$.

For each $(i, j)$, with $2 \leq i \leq k$ and $0 \leq j \leq \mu_{i}, \tilde{\psi}_{i-1, j}$ corresponds to right multiplication by some element $h_{i j}$ of $\mathscr{H}$. Also, $\theta$ corresponds to right multiplication by some element $h_{\theta}$ of $\mathscr{H}$ (see Lemma 6.7(i)). Now,

$$
\bigcap_{i=2}^{k} \bigcap_{j=0}^{\mu_{i}-1}\left\{h y_{\mu} \mid h \in \mathscr{H} \text { and } h y_{\mu} h_{i j}=0\right\} \subseteq\left\{h y_{\mu} \mid h \in \mathscr{H} \text { and } h y_{\mu} h_{\theta}=0\right\},
$$

by the version of Theorem 3.6 which is obtained by replacing $x_{\mu} \mathscr{H}$ by $\mathscr{H} y_{\mu}$. If $X$ is a left ideal of $\mathscr{H}$, then we denote the right annihilator $\{h \in \mathscr{H} \mid X h=0\}$ of $X$ by $r_{\mathscr{H}}(X)$. By taking the right annihilators of the two left ideals of $\mathscr{H}$ which are given above, we see that $h_{\theta} \in \sum_{i, j} h_{i j} \mathscr{H}+r_{\mathscr{H}}\left(\mathscr{H} y_{\mu}\right)$. Therefore, $v \theta=v h_{\theta}=0$, as we wished to show.

7.5. Lemma. We have $K^{\mu} \geq L^{\mu} \cap \operatorname{lr}\left(z_{\mu^{\prime}}\right) \geq W^{\mu^{\prime}}$.

Proof. Obviously, $L^{\mu} \cap \operatorname{lr}\left(z_{\mu^{\prime}}\right) \geq W^{\mu^{\prime}}$.

Assume that $\mu q \lambda$ and that $\theta \in \operatorname{Hom}_{S_{R}(n, r)}\left(L^{\mu}, L^{\lambda}\right)$. Then $\theta$ is right multiplication by $h \in \mathscr{H}$, say, in view of Lemma 6.7. Now, $z_{\mu^{\prime}} h=z_{\mu^{\prime}} \theta=0$, by Lemma 4.8. Therefore, if $v \in L^{\mu} \cap \operatorname{lr}\left(z_{\mu^{\prime}}\right)$ then $v \theta=v h=0$. That is, $L^{\mu} \cap \operatorname{lr}\left(z_{\mu^{\prime}}\right) \leq \bar{K}^{\mu}=K^{\mu}$, as required.

In order to obtain the equality of $K^{\mu}=W^{\mu^{\prime}}$, we shall prove that $\operatorname{dim} K^{\mu} \leq$ $\operatorname{dim} W^{\mu^{\prime}}$ when $R$ is a field. To facilitate the proof of this inequality, we reintroduce from [2] some notation concerning tableaux.

If $\lambda \models r$, then let $t^{\lambda}$ be the $\lambda$-tableau in which the numbers $1,2, \ldots, r$ appear in order along the rows. The group $\mathfrak{S}_{r}$ acts on the set of $\lambda$-tableaux by letter permutations. This lets us interpret our earlier definitions of $\mathscr{D}_{\lambda}$ and of $w_{\lambda}$ as follows. The permutation $d$ belongs to $\mathscr{D}_{\lambda}$ if and only if $t^{\lambda} d$ is row-standard (that is, it's entries increase along the rows). The permutation $w_{\lambda}$ is that element of $\mathfrak{S}_{r}$ which as the property that $1,2, \ldots, r$ appear in order along the columns of $t^{\lambda} w_{\lambda}$.

Now suppose that $\mu$ and $\lambda=\left(\lambda_{1}, \lambda_{2}, \ldots\right)$ are compositions of $r$. A $\mu$ tableau of type $\lambda$ is a $\mu$-tableau with (possibly) repeated entries, where for each $i$, the number of entries $i$ is equal to $\lambda_{i}$. We denote the set of $\mu$-tableaux of type $\lambda$ by $T(\mu, \lambda)$. For $A \in T(\mu, \lambda)$, we say that $A$ is row-standard if the numbers are nondecreasing along each row of $A$ and strictly row-standard if the numbers are increasing along each row of $A$. We define column-standard and strictly column-standard similarly. The tableau $A$ is semistandard if it is row-standard and strictly column-standard. Let $T_{0}(\mu, \lambda)$ denote the set of semistandard $\mu$-tableaux of type $\lambda$. 
Our first aim is to show that $\operatorname{dim}\left(K^{\mu^{\prime}} \cap M^{\lambda}\right) \leq\left|T_{0}(\mu, \lambda)\right|$ and we shall later demonstrate that $\operatorname{dim}\left(W^{\mu} \cap M^{\lambda}\right) \geq\left|T_{0}(\mu, \lambda)\right|$. These inequalities will prove that $\operatorname{dim} K^{\mu^{\prime}} \leq \operatorname{dim} W^{\mu}$.

We begin by giving an alternative description of $\mathscr{C}_{\lambda \mu}$. Recall that $\mathscr{C}_{\lambda \mu}$ is defined to be the subset of $\mathscr{D}_{\lambda \mu}=\mathscr{D}_{\lambda} \cap \mathscr{D}_{\mu}^{-1}$ consisting of those elements $d$ such that $\mathfrak{S}_{\lambda} \cap d \mathfrak{S}_{\mu} d^{-1}=(1)$. We have seen that $\mathscr{C}_{\lambda \mu}$ indexes a basis of $L^{\mu} \cap M^{\lambda}$.

7.6. Lemma. Suppose that $\lambda, \mu \in \Lambda(n, r)$. Then $\mathscr{C}_{\lambda \mu}$ is the set of elements $d$ in $\mathscr{D}_{\lambda}$ which have the property that whenever $i<j$ and $i$ and $j$ belong to the same row of $t^{\mu}$, then the row index of $i$ in $t^{\lambda} d$ is less than the row index of $j$ in $t^{\lambda} d$.

Proof. Let $C$ denote the set of elements $d$ in $\mathscr{D}_{\lambda}$ which have the property that if $i$ and $i+1$ belong to the same row of $t^{\mu}$ then the row index of $i$ in $t^{\lambda} d$ is less than the row index of $i+1$ in $t^{\lambda} d$. We must prove that $C=\mathscr{C}_{\lambda \mu}$. Throughout the proof, we assume that $i$ and $i+1$ belong to the same row of $t^{\mu}$ and denote $(i, i+1)$ by $a$. Thus, $a$ is a basic transposition in $\mathfrak{S}_{\mu}$.

Let $d \in \mathscr{C}_{\lambda \mu}$. Then $d \in \mathscr{D}_{\lambda}$ and $\left|\mathfrak{S}_{\lambda} d \mathfrak{S}_{\mu}\right|=\left|\mathfrak{S}_{\lambda}\right|\left|\mathfrak{S}_{\mu}\right|$. Hence $d \mathfrak{S}_{\mu} \subseteq \mathscr{D}_{\lambda}$, so $d a \in \mathscr{D}_{\lambda}$ and $l(d a)=l(d)+1$. Therefore, the row index of $i$ in $t^{\lambda} d$ is less than the row index of $i+1$ in $t^{\lambda} d$, so $d \in C$. We have now proved that $\mathscr{C}_{\lambda \mu} \subseteq C$.

Next, let $d \in C$. Then $d \in \mathscr{D}_{\lambda}$ and the row index of $i$ in $t^{\lambda} d$ is less than that of $i+1$, so $d a \in \mathscr{D}_{\lambda}$ and $l(d a)=l(d)+1$. Since $l(d a)=l(d)+1$ for all basic transpositions $a$ in $\mathfrak{S}_{\mu}$, we deduce that $d$ is a distinguished left coset representative of $\mathfrak{S}_{\mu}$, so $d \in \mathscr{D}_{\mu}^{-1}$. Since $d a \in \mathscr{D}_{\lambda}$ and $d \in \mathscr{D}_{\lambda}$, we have dad $^{-1} \notin \mathfrak{S}_{\lambda}$. Thus, dad $^{-1} \notin \mathfrak{S}_{\lambda}$ for all basic transpositions $a$ in $\mathfrak{S}_{\mu}$, and henc $\mathfrak{S}_{\lambda} \cap d \mathfrak{S}_{\mu} d^{-1}=(1)$. This shows that $C \subseteq \mathscr{C}_{\lambda \mu}$ and completes the proof of the lemma.

From here until the end of this section, fix $\lambda \in \Lambda(n, r)$ and $\mu \vdash r$. (Remember that we are assuming that $n \geq r$, so $\mu \in \Lambda(n, r)$.)

7.7. Definition. If $d \in \mathscr{D}_{\lambda}$ then define $A_{d} \in T(\mu, \lambda)$ by specifying that for all $a$ and $b$ if the entry in row $a$ and column $b$ of $t^{\mu} w_{\mu}$ is $i$, then the entry in row $a$ and column $b$ of $A_{d}$ is the row index of $i$ in $t^{\lambda} d$.

7.8. Example. Suppose that $\mu=\left(42^{2}\right), \lambda=\left(32^{2} 1\right)$, and

$$
t^{\lambda} d=\begin{array}{ll}
1 & 27 \\
3 & 5 \\
6 & 8 \\
4
\end{array}
$$


(Notice that $t^{\lambda} d$ is row-standard, so $d \in \mathscr{D}_{\lambda}$.) We have

$$
t^{\mu} w_{\mu}=\begin{aligned}
& 1478 \\
& 25 \\
& 36
\end{aligned}
$$

and $A_{d}$ is obtained from $t^{\mu} w_{\mu}$ simply by replacing each number by its corresponding row index in $t^{\lambda} d$ :

$$
A_{d}=\begin{array}{lll}
1413 \\
12 \\
23
\end{array}
$$

It is easy to see that the map $d \mapsto A_{d}$ is a bijection between $\mathscr{D}_{\lambda}$ and $T(\mu, \lambda)$. (In fact, the inverse map is $A \mapsto\left(w_{\mu}\right)_{A}$, given by Definition 7.20 below.)

In view of Lemma 7.6, we have

7.9. Lemma. The bijection $d \mapsto A_{d}$ between $\mathscr{D}_{\lambda}$ and $T(\mu, \lambda)$, upon restriction to $\mathscr{C}_{\lambda \mu^{\prime}}$ gives a bijection between $\mathscr{C}_{\lambda \mu^{\prime}}$ and the set of strictly column-standard $\mu$-tableaux of type $\lambda$.

We remark, in passing, that this demonstrates that the rank of the free $R$ module $L^{\mu^{\prime}}$ is equal to the number of strictly column-standard tableaux in $T(\mu, \lambda)$. The next lemma, which is similar in style to an argument of Carter and Lusztig [1, §3], will furnish us with the inequality $\operatorname{dim}\left(K^{\mu^{\prime}} \cap M^{\lambda}\right) \geq\left|T_{0}(\mu, \lambda)\right|$.

7.10. Lemma. Suppose that $\mu \vdash r$ and that $\lambda \in \Lambda(n, r)$. Assume that $v \in$ $K^{\mu^{\prime}} \cap M^{\lambda}$. Then $v=\sum_{d \in \mathscr{C}_{\lambda \mu^{\prime}}} c_{d} \phi_{\lambda \omega}^{d} y_{\mu^{\prime}}$ for some $c_{d} \in R$. If $v \neq 0$, then for some $e \in \mathscr{C}_{\lambda \mu^{\prime}}$ which corresponds, as in Lemma 7.9, to a semistandard $\mu$-tableau $A_{e}$ of type $\lambda$, we have $c_{e} \neq 0$.

Proof. Define a relation $\unrhd$ on the set of $\mu$-tableaux of type $\lambda$ by specifying that $A \unrhd B$ if for all $j$ the sum of the numbers in the first $j$ columns of $A$ is at most the sum of the numbers in the first $j$ columns of $B$. Write $A \triangleright B$ if we have strict inequality somewhere.

If $d \in \mathscr{C}_{\lambda \mu^{\prime}}$ then let $A_{d}$ be the corresponding strictly column-standard $\mu$ tableau of type $\lambda$ (see Lemma 7.9).

Now, $v$ can be written in the form stated in the lemma, in view of Theorem 5.2. Assume that $v \neq 0$. Choose $e=\mathscr{C}_{\lambda \mu^{\prime}}$ such that $c_{e} \neq 0$ but $c_{d}=0$ if $A_{d} \triangleright A_{e}$. We aim to show that $A_{e}$ is semistandard.

Since $A_{e}$ is strictly column-standard, we shall be home if we get a contradiction from assuming that two adjacent columns of $A_{e}$, say column $j$ and column $j+1$, have entries $a_{1}<a_{2}<\cdots<a_{m}$ and $b_{1}<b_{2}<\cdots<b_{m}$ 
respectively, and $a_{z}>b_{z}$ for some $z$.

$$
\begin{array}{ccc}
a_{1} & b_{1} \\
\wedge & \wedge \\
\vdots & \vdots \\
A_{e}: & \hat{a_{z}} & \multicolumn{1}{c}{} \\
\hat{b} & \wedge \\
\vdots & \vdots \\
\vdots & \wedge \\
\wedge & b_{n} \\
a_{m} &
\end{array}
$$

Let $d_{1}, d_{2}, \ldots, d_{m}$ be the entries in column $j$ of $t^{\mu} w_{\mu}$ and let $d_{m+1}, d_{m+2}$, $\ldots, d_{m+n}$ be the entries in column $j+1$ of $t^{\mu} w_{\mu}$. (Note that $d_{1+i}=d_{1}+i$ for all $i$.)

Let $X^{\prime}=\left\{d_{1}, \ldots, d_{z-1}\right\}, X=\left\{d_{z}, \ldots, d_{m}\right\}, Y=\left\{d_{m+1}, \ldots, d_{m+z}\right\}$, and $Y^{\prime}=\left\{d_{m+z+1}, \ldots, d_{m+n}\right\}$.

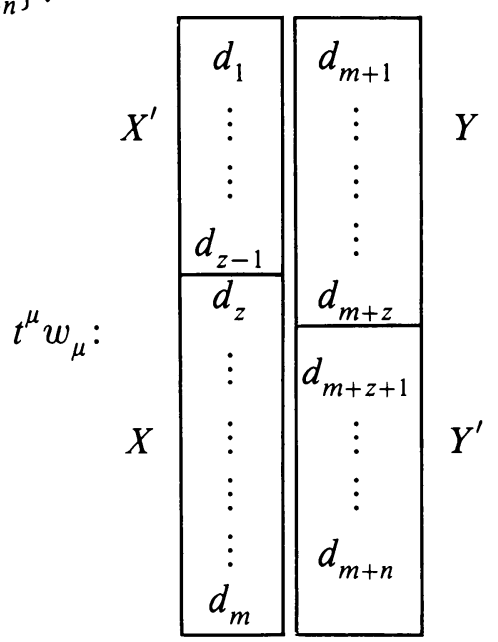

If $Z$ is any subset of $\{1,2, \ldots, r\}$ then let $\mathfrak{S}_{Z}$ denote the symmetric group on $Z$ and let $y_{Z}=\sum_{w \in \mathfrak{S}_{Z}}(-q)^{-l(w)} T_{w}$.

Note that $y_{X} y_{Y} h=y_{X \cup Y}$ for some $h \in \mathscr{C}$ and $h_{1} y_{X^{\prime}} y_{Y^{\prime}} y_{X} y_{Y}=y_{X \cup X^{\prime}} y_{Y \cup Y^{\prime}}$ for some $h_{1} \in \mathscr{H}$. Hence $y_{X \cup X^{\prime}} y_{Y \cup Y^{\prime}} h=h_{1} y_{X^{\prime}} y_{Y^{\prime}} y_{X} y_{Y} h=h_{1} y_{X \cup Y} y_{X^{\prime}} y_{Y^{\prime}}$. Therefore, $y_{\mu^{\prime}} h=h_{1} y_{\nu}$, where

$$
\nu=\left(\mu_{1}^{\prime}, \mu_{2}^{\prime}, \ldots, \mu_{j-1}^{\prime},\left|X^{\prime}\right|,|X \cup Y|,\left|Y^{\prime}\right|, \mu_{j+2}^{\prime}, \ldots\right) .
$$

Now, postmultiplication by $h$ gives an $S_{R}(n, r)$-homomorphism from $L^{\mu^{\prime}}$ to $L^{\nu}$. But $v \in K^{\mu^{\prime}}=\bar{K}^{\mu^{\prime}}$ (see Lemma 7.4), and $\mu^{\prime} \notin \nu$. Therefore, $v h=0$.

Since $v \in M^{\lambda}$, we know that $v$ is a linear combination of terms $\phi_{\lambda \omega}^{d}$, where $d \in \mathscr{D}_{\lambda}$. We write $v=v_{1}+v_{2}$, where $v_{1}$ is a linear combination of terms 
$\phi_{\lambda \omega}^{d}$ with $d \in \mathscr{D}_{\lambda}$ and $d=e w$ for some $w \in \mathfrak{S}_{X \cup Y}$, where $v_{2}$ is a linear combination of terms $\phi_{\lambda w}^{d}$ with $d \in \mathfrak{D}_{\lambda}$, and where $d$ is not of the form ew with $w \in \mathfrak{S}_{X \cup Y}$. We shall deduce $v_{1} h=0$ from the fact that $v h=0$.

Now, since $b_{1}<b_{2}<\cdots<b_{z}<a_{z}<\cdots<a_{m}$, we have $e w \in \mathscr{D}_{\lambda}$ and $\phi_{\lambda \omega}^{e} T_{w}=\phi_{\lambda \omega}^{e w}$ for all $w \in \mathfrak{S}_{X \cup Y}$. Hence, if $u, w \in \mathfrak{S}_{X \cup Y}$ then $\phi_{\lambda \omega}^{e w} T_{u}$ is a linear combination of terms $\phi_{\lambda \omega}^{e w^{\prime}}$ with $w^{\prime} \in \mathfrak{S}_{X \cup Y}$.

On the other hand, suppose that $d \in \mathscr{D}_{\lambda}$ but $d$ cannot be written in the form $e w$ with $w \in \mathfrak{S}_{X \cup Y}$. In this case, for each $u \in \mathfrak{S}_{X \cup Y}$, we see that $\phi_{\lambda \omega}^{d} T_{u}$ does not involve any term of the form $\phi_{\lambda \omega}^{e w^{\prime}}$ with $w^{\prime} \in \mathfrak{S}_{X \cup Y}$, as can easily be proved by induction on $l(u)$, using result $(2.11)$.

Since $v_{1} h+v_{2} h=0$, and $h$ is a linear combination of terms $T_{u}$ with $u \in \mathfrak{S}_{X \cup Y}$, the results of the last two paragraphs show that $v_{1} h=0$.

Finally, we work out what $v_{1}$ must be. If $w \in \mathfrak{S}_{X \cup Y} \backslash\left(\mathfrak{S}_{X} \times \mathfrak{S}_{Y}\right)$ then $A_{e w} \triangleright A_{e}$, so $c_{e w}=0$, by our choice of $e$. Thus, $v_{1}$ is that part of $v$ which is a combination of terms $\phi_{\lambda \omega}^{d}$ with $d \in e\left(\mathfrak{S}_{X} \times \mathfrak{S}_{Y}\right)$. Therefore, $v_{1}=c_{e} \sum_{w \in \mathfrak{S}_{X} \times \mathfrak{S}_{Y}}(-q)^{-l(w)} \phi_{\lambda \omega}^{e} T_{w}=c_{e} \phi_{\lambda \omega}^{e} y_{X} y_{Y}$. (Recall that $\phi_{\lambda \omega}^{e w}=\phi_{\lambda \omega}^{e} T_{w}$ if $\left.w \in \mathfrak{S}_{X \cup Y}.\right)$

Since $v_{1} h=0$ and $c_{e} \neq 0$ and $y_{X} y_{Y} h=y_{X \cup Y}$, we have $\phi_{\lambda \omega}^{e} y_{X \cup Y}=0$. But

$$
\phi_{\lambda \omega}^{e} y_{X \cup Y}=\sum_{w \in \mathfrak{S}_{X \cup Y}}(-q)^{-l(w)} \phi_{\lambda \omega}^{e} T_{w}=\sum_{w \in \mathfrak{S}_{X \cup Y}}(-q)^{-l(w)} \phi_{\lambda \omega}^{e w},
$$

which is nonzero. This is the desired contradiction, and the lemma is proved.

7.11. Corollary. Suppose that $\mu \vdash r$ and that $\lambda \in \Lambda(n, r)$. Assume that $R$ is a field. Then the dimension of $K^{\mu^{\prime}} \cap M^{\lambda}$ is at most the number of semistandard $\mu$-tableaux of type $\lambda$.

Proof. The corollary follows from the lemma by elementary linear algebra (see $[3,2.13])$.

As we said earlier, we want to prove that $\operatorname{dim}\left(K^{\mu^{\prime}} \cap M^{\lambda}\right) \leq\left|T_{0}(\mu, \lambda)\right| \leq$ $\operatorname{dim}\left(W^{\mu} \cap M^{\lambda}\right)$. Corollary 7.11 establishes the first of these inequalities, and we now begin the task of proving the second inequality. Several preliminary definitions and results are needed.

Let $t_{1}$ be a $\lambda$-tableau and $t_{2}$ be a $\mu$-tableau. (We reserve lower case symbols like $t_{i}$ for tableaux whose entries are $1,2, \ldots, r$, without repeats.) We wish to define an $n$-by- $n$ matrix $\chi\left(t_{1}, t_{2}\right)$ which depends on $t_{1}$ and $t_{2}$. Remember that both $\lambda$ and $\mu$ have precisely $n$ parts, some of which might be zero.

7.12. Definition. Suppose that $t_{1}$ is a $\lambda$-tableau and $t_{2}$ is a $\mu$-tableau. Let $\chi\left(t_{1}, t_{2}\right)$ be the $n$-by- $n$ matrix whose entry in row $i$ and column $j$ is the cardinality of

\{entries in the first $i$ rows of $\left.t_{1}\right\} \cap$ \{entries in the first $j$ columns of $\left.t_{2}\right\}$. 
7.13. Example. Suppose that $n=r=9$ and

$$
\begin{aligned}
& 97425 \quad 3259 \\
& t_{1}=\begin{array}{ll}
138 \\
6
\end{array} \quad t_{2}=\begin{array}{ll}
186 \\
74
\end{array}
\end{aligned}
$$

Then $\chi\left(t_{1}, t_{2}\right)$ is the $9 \times 9$ matrix:

$$
\left(\begin{array}{llllll}
1 & 3 & 4 & 5 & 5 & \cdots \\
3 & 6 & 7 & 8 & 8 & \cdots \\
3 & 6 & 8 & 9 & 9 & \\
3 & 6 & 8 & 9 & 9 & \\
3 & 6 & 8 & 9 & 9 & \\
\vdots & \vdots & & & &
\end{array}\right) .
$$

If $t_{1}$ and $t_{1}^{\prime}$ are $\lambda$-tableaux and $t_{2}$ and $t_{2}^{\prime}$ are $\mu$-tableaux, then write $\chi\left(t_{1}, t_{2}\right) \geq \chi\left(t_{1}^{\prime}, t_{2}^{\prime}\right)$ if each entry in $\chi\left(t_{1}, t_{2}\right)$ is at least as big as the corresponding entry in $\chi\left(t_{1}^{\prime}, t_{2}^{\prime}\right)$. Write $\chi\left(t_{1}, t_{2}\right)>\chi\left(t_{1}^{\prime}, t_{2}^{\prime}\right)$ if, in addition, $\chi\left(t_{1}, t_{2}\right) \neq \chi\left(t_{1}^{\prime}, t_{2}^{\prime}\right)$.

The following properties of our matrices are immediate from the definitions.

$$
\chi\left(t_{1} w, t_{2} w\right)=\chi\left(t_{1}, t_{2}\right) \text { for all } w \in \mathfrak{S}_{r} .
$$

(i) $\chi\left(t_{1} w, t_{2}\right)=\chi\left(t_{1}, t_{2}\right)$ if $w$ belongs to the row stabilizer of $t_{1}$.

(ii) $\chi\left(t_{1}, t_{2} w\right)=\chi\left(t_{1}, t_{2}\right)$ if $w$ belongs to the column stabilizer of $t_{2}$.

If $1 \leq i \leq r$ and $1 \leq j \leq r$ and the row index of $i$ in $t_{1}$ is less than the row index of $j$ in $t_{1}$ and the column index of $i$ in $t_{2}$ is less than the column index of $j$ in $t_{2}$, then $\chi\left(t_{1}, t_{2}\right)>\chi\left(t_{1}(i, j), t_{2}\right)=\chi\left(t_{1}, t_{2}(i, j)\right)$.

We are going to construct linearly independent elements of $W^{\mu} \cap M^{\lambda}$, and we begin by doing some calculations with $M^{\lambda}$. Remember that $\left\{\phi_{\lambda \omega}^{d} \mid d \in \mathscr{D}_{\lambda}\right\}$ is a basis of $M^{\lambda}$. We translate result (2.11) concerning products $\phi_{\lambda \omega}^{d} T_{d}$ into a form which is more convenient for our current calculations. If $w \in \mathfrak{S}_{r}$, then we denote by $\bar{w}$ the unique element of $\mathfrak{S}_{\lambda} w \cap \mathscr{D}_{\lambda}$.

7.17. Lemma. Suppose that $d \in \mathscr{D}_{\lambda}$ and that $a=(i, i+1)$ is a basic transposition in $\mathfrak{S}_{r}$. Then

$$
\phi_{\lambda \omega}^{d} T_{a}=q^{\delta} \phi_{\lambda \omega}^{\overline{d a}}+c \phi_{\lambda \omega}^{d},
$$

where $\delta=0$ or 1 and $c=q-1$ if the row index of $i+1$ in $t^{\lambda} d$ is less than the row index of $i$ in $t^{\lambda} d$, and $c=0$, otherwise.

Proof. Result (2.11) shows that if $i+1$ belong to the same row of $t^{\lambda} d$ then $\phi_{\lambda \omega}^{d} T_{a}=q \phi_{\lambda \omega}^{d}=q \phi_{\lambda \omega}^{\overline{d a}}$, and if the row index of $i$ in $t^{\lambda} d$ is less than that of 
$i+1$ then $\phi_{\lambda \omega}^{d} T_{a}=\phi_{\lambda \omega}^{d a}=\phi_{\lambda \omega}^{\overline{d a}}$, and if the row index of $i+1$ in $t^{\lambda} d$ is less than that of $i$ then $\phi_{\lambda \omega}^{d} T_{a}=q \phi_{\lambda \omega}^{d a}+(q-1) \phi_{\lambda \omega}^{d}=q \phi_{\lambda \omega}^{\overline{d a}}+(q-1) \phi_{\lambda \omega}^{d}$.

7.18. Lemma. Suppose that $u$ and $w \in \mathfrak{S}_{r}$ and that $t^{\mu} w$ is standard. Then for some integer $k$, and some $s \in M^{\lambda}$, we have

(i) $\phi_{\lambda \omega}^{1} T_{u} T_{w}=q^{k} \phi_{\lambda \omega}^{\overline{u w}}+s$, and

(ii) $s$ is a linear combination of terms $\phi_{\lambda \omega}^{d}\left(d \in \mathscr{D}_{\lambda}\right)$ for which $\chi\left(t^{\lambda} u, t^{\mu}\right)>$ $\chi\left(t^{\lambda} d, t^{\mu} w\right)$.

Proof. The conclusion of the lemma is correct when $w=1$, so assume that $w \neq 1$. Then for some $w^{\prime} \in \mathfrak{S}_{r}$ and some $a=(i, i+1) \in \mathfrak{S}_{r}$, we have that $w=w^{\prime} a$, and $t^{\mu} w^{\prime}$ is standard, and the column index of $i+1$ in $t^{\mu} w^{\prime}$ is less than the column index of $i$ in $t^{\mu} w^{\prime}$. By induction, we have

$$
\phi_{\lambda \omega}^{1} T_{u} T_{w^{\prime}}=q^{k^{\prime}} \phi_{\lambda \omega}^{\overline{u w^{\prime}}}+s^{\prime},
$$

where $k^{\prime}$ is an integer and $s^{\prime}$ is a linear combination of terms $\phi_{\lambda \omega}^{d} \quad\left(d \in \mathscr{D}_{\lambda}\right)$ for which $\chi\left(t^{\lambda} u, t^{\mu}\right)>\chi\left(t^{\lambda} d, t^{\mu} w^{\prime}\right)$.

We multiply the above equation on the right by $T_{a}$. Of course, $T_{w^{\prime}} T_{a}=T_{w}$, so we get $\phi_{\lambda \mu}^{1} T_{u} T_{w}$ on the left-hand side. We check that every term $\phi_{\lambda \omega}^{d}$ in $\left(q^{k^{\prime}} \phi_{\lambda \omega}^{\overline{u w^{\prime}}}+s^{\prime}\right) \underline{T_{a}}$, except $\phi_{\lambda \omega}^{\overline{u w}}$, satisfies $\chi\left(t^{\lambda} u, t^{\mu}\right)>\chi\left(t^{\lambda} \frac{\left.d, t^{\mu} w\right)}{u w^{\prime}}\right.$.

Consider $\phi_{\lambda \omega}^{\overline{u w^{\prime}}} T_{a}$. By Lemma 7.17 and result (7.16), $\phi_{\lambda \omega}^{\overline{u w^{\prime}}} T_{a}=q^{\delta} \phi_{\lambda \omega}^{\overline{u w}}+c \phi_{\lambda \omega}^{\overline{u \omega^{\prime}}}$ and if $c \neq 0$ then $\chi\left(t^{\lambda} \overline{u w^{\prime}}, t^{\mu} w^{\prime}\right)>\chi\left(t^{\lambda} \overline{u w^{\prime}}, t^{\mu} w\right)$. But $\chi\left(t^{\lambda} \overline{u w^{\prime}}, t^{\mu} w^{\prime}\right)=$ $\chi\left(t^{\lambda} u w^{\prime}, t^{\mu} w^{\prime}\right)=\chi\left(t^{\lambda} u, t^{\mu}\right)$, by results (7.15)(i) and (7.14). Thus, if $\overline{\phi_{\lambda \omega}^{\overline{w w^{\prime}}}}$ occurs in the product $\phi_{\lambda \omega}^{\overline{u w^{\prime}}} T_{a}$ then $\chi\left(t^{\lambda} u, t^{\mu}\right)>\chi\left(t^{\lambda} \overline{u w^{\prime}}, t^{\mu} w\right)$.

Next we turn our attention to $s^{\prime} T_{a}$. Suppose that $\phi_{\lambda \omega}^{d}$ is one of the terms in $s^{\prime}$. Then $\chi\left(t^{\lambda} u, t^{\mu}\right)>\chi\left(t^{\lambda} d, t^{\mu} w^{\prime}\right)$ and from Lemma 7.17, $\phi_{\lambda \omega}^{d} T_{a}=$ $q^{\varepsilon} \phi_{\lambda \omega}^{\overline{d a}}+c \phi_{\lambda \omega}^{d}$. We have $\chi\left(t^{\lambda} u, t^{\mu}\right)>\chi\left(t^{\lambda} d, t^{\mu} w^{\prime}\right)=\chi\left(t^{\lambda} \overline{d a}, t^{\mu} w\right)$, by applying results (7.14) and (7.15)(i). If $c \neq 0$, then result (7.16) gives $\chi\left(t^{\lambda} d, t^{\mu} w^{\prime}\right)>$ $\chi\left(t^{\lambda} d, t^{\mu} w\right)$, so $\chi\left(t^{\lambda} u, t^{\mu}\right)>\chi\left(t^{\lambda} d, t^{\mu} w\right)$. The proof of the lemma is now complete.

7.19. Lemma. Suppose that $u \in \mathfrak{S}_{r}$ and $w \in \mathfrak{S}_{\mu^{\prime}}$. Then $\phi_{\lambda \omega}^{1} T_{u} T_{w}$ is a linear combination of terms $\phi_{\lambda \omega}^{d}\left(d \in \mathscr{D}_{\lambda}\right)$ for which $\chi\left(t^{\lambda} d, t^{\mu} w_{\mu}\right)=\chi\left(t^{\lambda} u, t^{\mu} w_{\mu}\right)$.

Proof. The proof of this result is similar to the proof of Lemma 7.18, but is easier. We leave the details to the reader.

Next, we recall some combinatorial definitions and results from $[2, \S 1]$.

7.20. Definition. If $w \in \mathfrak{S}_{r}$ and $A \in T(\mu, \lambda)$ then let $w_{A} \in \mathscr{D}_{\lambda}$ be defined by letting $t^{\lambda} w_{A}$ be the row-standard $\lambda$-tableau for which $i$ belongs to row $a$ if the place occupied by $i$ in $t^{\mu} w$ is occupied by $a$ in $A$ (for all $i$ with $1 \leq i \leq r$ ). 
The following results are easy (see $[2,1.7])$.

(7.21) The map $A \mapsto 1_{A}$ gives a bijection between $T(\mu, \lambda)$ and $\mathscr{D}_{\lambda}$, and the restriction of this map to the set of row-standard tableau in $T(\mu, \lambda)$ maps onto $\mathscr{D}_{\lambda \mu}$.

(7.22) If $A, B \in T(\mu, \lambda)$ we write $A \sim B$ if $A$ and $B$ are row equivalent. Then $\mathfrak{S}_{\lambda} 1_{A} \mathfrak{S}_{\mu}=\bigcup_{B \sim A} \mathfrak{S}_{\lambda} 1_{B}$ (disjoint union).

(7.23) If $A \in T(\mu, \lambda)$ and $w \in \mathfrak{S}_{r}$ then $w_{A}$ is the unique element in $\mathfrak{S}_{\lambda} 1_{A} w \cap \mathscr{D}_{\lambda}$.

(7.24) If $A$ and $B$ are distinct elements of $T(\lambda, \mu), B$ is row equivalent to $A$, and $A$ is row-standard, then $\chi\left(t^{\lambda} 1_{A}, t^{\mu}\right)>\chi\left(t^{\lambda} 1_{B}, t^{\mu}\right)$.

(7.25) If $A$ and $B$ are distinct elements of $T_{0}(\mu, \lambda)$ then $\chi\left(t^{\mu}, t^{\lambda} 1_{A}\right) \neq$ $\chi\left(t^{\mu}, t^{\lambda} 1_{B}\right)$.

7.26. Lemma. Suppose that $A \in T(\mu, \lambda)$ and that $A$ is row-standard. Then for some integer $k$, and some $s \in M^{\lambda}$, we have

(i) $\phi_{\lambda \mu}^{1} \phi_{\mu \omega}^{1} T_{w_{\mu}}=q^{k} \phi_{\lambda \omega}^{1} T_{\left(w_{\mu}\right)_{A}}+s$, and

(ii) $s$ is a linear combination of terms $\phi_{\lambda \omega}^{d}\left(d \in \mathscr{D}_{\lambda}\right)$ for which $\chi\left(t^{\lambda} 1_{A}, t^{\mu}\right)>$ $\chi\left(t^{\lambda} d, t^{\mu} w_{\mu}\right)$.

Proof. From result (7.22) we obtain

$$
\phi_{\lambda \mu}^{1_{A}^{A}} \phi_{\mu \omega}^{1}=\sum_{B \sim A} \phi_{\lambda \omega}^{1_{B}}
$$

Now, by Lemma 7.18

$$
\phi_{\lambda \omega}^{1} T_{w_{\mu}}=q^{k_{B}} \phi_{\lambda \omega}^{1} T_{\overline{1_{B} w_{\mu}}}+s_{B},
$$

where $k_{B}$ is an integer and $s_{B}$ is a linear combination of terms $\phi_{\lambda \omega}^{d}$ for which $\chi\left(t^{\lambda} 1_{B}, t^{\mu}\right)>\chi\left(t^{\lambda} d, t^{\mu} w_{\mu}\right)$. Moreover, $\chi\left(t^{\lambda} 1_{A}, t^{\mu}\right)>\chi\left(t^{\lambda} 1_{B}, t^{\mu}\right)=$ $\chi\left(t^{\lambda} \overline{1}_{B} w_{\mu}, t^{\mu} w_{\mu}\right)$ if $B \sim A$ but $B \neq A$, by result (7.24). Hence

$$
\phi_{\lambda \mu}^{1 A} \phi_{\mu \omega}^{1} T_{w_{\mu}}=q^{k} \phi_{\lambda \omega}^{1} T_{\overline{1_{A} w_{\mu}}}+s,
$$

where $k$ is an integer and $s$ is a linear combination of terms $\phi_{\lambda \omega}^{d}$ for which $\chi\left(t^{\lambda} 1_{A}, t^{\mu}\right)>\chi\left(t^{\lambda} d, t^{\mu} w_{\mu}\right)$. Now, $\overline{1}_{A} w_{\mu}=\left(w_{\mu}\right)_{A}$ by result (7.23), so the lemma is proved.

7.27. Theorem. Suppose that $\lambda \in \Lambda(n, r)$ and $\mu \vdash r$. Then $\left\{\phi_{\lambda \mu}^{1_{A}} z_{\mu} \mid A \in\right.$ $\left.T_{0}(\mu, \lambda)\right\}$ is a linearly independent subset of $W^{\mu} \cap M^{\lambda}$.

Proof. Suppose that $\sum c_{A} \phi_{\lambda \mu}^{1} z_{\mu}=0$, where $c_{A} \in R$ and the sum is over $A \in$ $T_{0}(\lambda, \mu)$. Choose $D \in T_{0}(\lambda, \mu)$ such that $c_{A}=0$ for all $A$ with $\chi\left(t^{\lambda} 1_{A}, t^{\mu}\right)>$ $\chi\left(t^{\lambda} 1_{D}, t^{\mu}\right)$. If we can prove that $c_{D}=0$ then it will follow that every coefficient $c_{A}=0$, and we will have finished. 
By Lemma 7.26 and result (7.25) there exists an integer $k$ and $s \in M^{\lambda}$ such that

$$
\sum c_{A} \phi_{\lambda \mu}^{1 A} \phi_{\mu \omega}^{1} T_{w_{\mu}}=c_{D} q^{k} \phi_{\lambda \omega}^{1} T_{\left(w_{\mu}\right)_{D}}+s
$$

where $s$ is a linear combination of terms $\phi_{\lambda \omega}^{d}\left(d \in \mathscr{D}_{\lambda}\right)$ for which $\chi\left(t^{\lambda} d, t^{\mu} w_{\mu}\right)$ $\ngtr \chi\left(t^{\lambda} 1_{D}, t^{\mu}\right)$.

Now,

$$
c_{D} q^{k} \phi_{\lambda \omega}^{1} T_{\left(w_{\mu}\right)_{D}} y_{\mu^{\prime}}+s y_{\mu^{\prime}}=\sum c_{A} \phi_{\lambda \mu}^{1} \phi_{\mu \omega}^{1} T_{w_{\mu}} y_{\mu^{\prime}}=0
$$

and by Lemma 7.19, $\phi_{\lambda \omega}^{1} T_{\left(w_{\mu}\right)_{D}} y_{\mu^{\prime}}$ is a linear combination of terms $\phi_{\lambda \omega}^{d} \quad(d \in$ $\left.\mathscr{D}_{\lambda}\right)$ for which $\chi\left(t^{\lambda} d, t^{\mu} w_{\mu}\right)=\chi\left(t^{\lambda}\left(w_{\mu}\right)_{D}, t^{\mu} w_{\mu}\right)=\chi\left(t^{\lambda} 1_{D}, t^{\mu}\right)$, while $s y_{\mu^{\prime}}$ is a linear combination of terms $\phi_{\lambda \omega}^{d}\left(d \in \mathscr{D}_{\lambda}\right)$ for which $\chi\left(t^{\lambda} d, t^{\mu} w_{\mu}\right) \neq$ $\chi\left(t^{\lambda} 1_{D}, t^{\mu}\right)$. Therefore, $c_{D} q^{k} \phi_{\lambda \omega}^{1} T_{\left(w_{\mu}\right)_{D}} y_{\mu^{\prime}}=0$. But $\phi_{\lambda \omega}^{1} T_{\left(w_{\mu}\right)_{D}} y_{\mu^{\prime}} \neq 0$, since the numbers strictly increase down the columns of $D$. Therefore, $c_{D}=0$, as we wished to show.

\section{The Main Results}

We are now able to show that $q$-Weyl modules are free as $R$-modules, and exhibit a basis. We can also classify the irreducible modules for $q$-Schur algebras (when $R$ is a field).

The following two theorems will be proved simultaneously.

8.1. Theorem (the Semistandard Basis Theorem for $q$-Weyl modules). Suppose that $\mu \vdash r$. Then the $q$-Weyl module $W^{\mu}$ is free as an $R$-module and

$$
\left\{\phi_{\lambda \mu}^{1} z_{\mu} \mid \lambda \in \Lambda(n, r) \text { and } A \in T_{0}(\mu, \lambda)\right\}
$$

is a basis. In particular, the rank of $W^{\mu}$ is equal to the number of semistandard $\mu$-tableaux.

8.2. Theorem (the Kernel Intersection Theorem for $q$-Weyl modules). Suppose that $\mu \vdash r$. Then $W^{\mu}=K^{\mu^{\prime}}$.

Proof of Theorems 8.1 and 8.2. Assume first that $R$ is a field. We have $K^{\mu^{\prime}} \cap$ $M^{\lambda} \geq W^{\mu} \cap M^{\lambda}$ by Lemma 4.8. But $\left|T_{0}(\mu, \lambda)\right| \geq \operatorname{dim}\left(K^{\mu^{\prime}} \cap M^{\lambda}\right)$ by Corollary 7.11 and $\left\{\phi_{\lambda \mu}^{1{ }^{A}} z_{\mu} \mid A \in T_{0}(\mu, \lambda)\right\}$ is a linearly independent subset of $W^{\mu} \cap M^{\lambda}$ by Theorem 7.27. Therefore, $K^{\mu^{\prime}} \cap M^{\lambda}=W^{\mu} \cap M^{\lambda}$ and $\left\{\phi_{\lambda \mu}^{1_{A} z_{\mu}} \mid A \in T_{0}(\mu, \lambda)\right\}$ is a basis of this space. The proof of the theorems in the case where $R$ is a field is now complete.

Next let $R$ be an integral domain, with quotient field $F$. Let $v \in K^{\mu^{\prime}} \cap M^{\lambda}$. By extending the ring of coefficients to $F$, we can write $v=\sum c_{A} \phi_{\lambda \mu}^{1_{A} z_{\mu}}$, where $c_{A} \in F$ and the sum is over $A \in T_{0}(\mu, \lambda)$. We prove that $c_{A} \in R$ for all $A$, by induction on the number of nonzero coefficients $c_{A}$. We may assume that 
$v \neq 0$. Choose $D \in T_{0}(\lambda, \mu)$ such that $c_{D} \neq 0$, but $c_{A}=0$ for all $A$ with $\chi\left(t^{\lambda} 1_{A}, t^{\mu}\right)>\chi\left(t^{\lambda} 1_{D}, t^{\mu}\right)$.

Precisely as in the proof of Theorem 7.27, there exists an integer $k$ such that

$$
v=c_{D} q^{k} \phi_{\lambda \omega}^{1} T_{\left(w_{\mu}\right)_{D}} y_{\mu^{\prime}}+s y_{\mu^{\prime}},
$$

where $s \in M^{\lambda}$ and $s y_{\mu^{\prime}}$ is a linear combination of terms $\phi_{\lambda \omega}^{d}\left(d \in \mathscr{D}_{\lambda}\right)$ for which $\chi\left(t^{\lambda} d, t^{\mu} w_{\mu}\right) \neq \chi\left(t^{\lambda} 1_{D}, t^{\mu}\right)$. Now, if $d=\left(w_{\mu}\right)_{D}$ then $\chi\left(t^{\lambda} d, t^{\mu} w_{\mu}\right)=$ $\chi\left(t^{\lambda} 1_{D}, t^{\mu}\right)$ and hence $\phi_{\lambda \omega}^{d}$ does not occur in $s y_{\mu^{\prime}}$. Therefore, for this choice of $d$, the coefficient of $\phi_{\lambda \omega}^{d}$ in $v$ is $c_{D} q^{k}$. Since $v \in S_{R}(n, r)$, we deduce that $c_{D} \in R$.

Now, by induction, all the coefficients $c_{A}$ in $v-c_{D} \phi_{\lambda \mu}^{1} z_{\mu}$ belong to $R$. Hence $v \in W^{\mu} \cap M^{\lambda}$ and $\left\{\phi_{\lambda \mu}^{1}{ }_{A}^{A} z_{\mu} \mid A \in T_{0}(\lambda, \mu)\right\}$ is a basis of $W^{\mu} \cap M^{\lambda}$.

8.3. Corollary. We have $W^{\mu}=\operatorname{lr}\left(z_{\mu}\right) \cap L^{\mu^{\prime}}$.

Proof. The corollary follows immediately from Theorem 8.2 and Lemma 7.5.

8.4. Corollary. Assume that $q+1 \neq 0$ or $\mu$ is 2-regular. Then $W^{\mu}=\operatorname{lr}\left(z_{\mu}\right)$.

Proof. Apply Theorem 6.13 and Corollary 8.3.

8.5. Corollary. Assume that $q+1 \neq 0$ or $\mu$ is 2-regular, and that $U$ is a right $\mathscr{H}$-submodule of q-tensor space. Then $W^{\mu} \cap U$ is canonically isomorphic to $\operatorname{Hom}_{\mathscr{H}}\left(S^{\mu}, U\right)$, as an R-module.

Proof. Apply Lemma 6.3(i) and Corollary 8.4.

By combining Corollary 8.5, Lemma 6.8(ii), and Corollary 8.4, we obtain the following generalization of a theorem of Carter and Lusztig [1, Theorem 3.7].

8.6. Corollary. Suppose that $\lambda, \mu \in \Lambda(n, r)$. Then there exist canonical $R$ injections

$$
\operatorname{Hom}_{S_{R}(n, r)}\left(W^{\lambda}, W^{\mu}\right) \underset{P}{\leftarrow} S^{\lambda} \cap W^{\mu} \underset{Q}{\rightarrow} \operatorname{Hom}_{\mathscr{P}}\left(S^{\mu}, S^{\lambda}\right)
$$

such that

(i) $P$ is an isomorphism, and

(ii) if $q+1 \neq 0$ or $\mu$ is 2-regular, then $Q$ is an isomorphism.

8.7. Corollary (the Semistandard Homomorphism Theorem). Suppose that $\lambda \in$ $\Lambda(n, r)$ and $\mu \vdash r$. Let $\mathscr{E}$ denote the set of $\mathscr{H}$-homomorphisms from $S^{\mu}$ into $M^{\lambda}$ which can be extended to an element of $\operatorname{Hom}_{\mathscr{H}}\left(M^{\mu}, M^{\lambda}\right)$. Then

$$
\left\{\phi_{\lambda \mu}^{1} \mid A \in T_{0}(\lambda, \mu)\right\}
$$

is a basis for $\mathscr{E}$. If, in addition, $q+1 \neq 0$ or $\mu$ is 2-regular, then $\mathscr{E}=$ $\operatorname{Hom}_{\mathscr{H}}\left(S^{\mu}, M^{\lambda}\right)$.

Proof. The corollary follows from Lemma 6.9, Theorem 8.1, and Corollary 8.4. 
8.8. Theorem. Suppose that $R$ is a field. Then $\left\{F^{\mu} \mid \mu \vdash r\right\}$ is a complete set of nonisomorphic irreducible modules for the q-Schur algebra $S_{R}(n, r)$.

Proof. In Corollary 4.11, we proved all parts of the theorem, except that the given set is complete.

Since every irreducible $S_{R}(n, r)$-module is a composition factor of $E_{R}(n, r)$, and $E_{R}(n, r)=L^{\left(1^{\prime}\right)}$, it is certainly sufficient to prove the following:

(*) If $\mu \in \Lambda(n, r)$ then every composition factor of $L^{\mu}$ is isomorphic to some $F^{\lambda}$ with $\lambda \vdash r$ and $\mu^{\prime} \unrhd \lambda$.

We assume that if $\mu^{\prime} \triangleright \nu^{\prime}$ then every composition factor of $L^{\nu}$ has the form $F^{\lambda}$ for some $\lambda$ with $\nu^{\prime} \triangleright \lambda$. (This assumption is empty if $\mu=(r)$.)

Since $W^{\mu^{\prime}}=K^{\mu}$, all composition factor of $L^{\mu} / W^{\mu^{\prime}}$ have the form $F^{\lambda}$ with $\mu^{\prime} \triangleright \lambda$. The contravariant dual of $W^{\mu^{\prime} \perp}$ is isomorphic to $L^{\mu} / W^{\mu^{\prime}}$. Now recall that each $F^{\lambda}$ is self-dual (see Theorem 4.6). Consider the following picture:

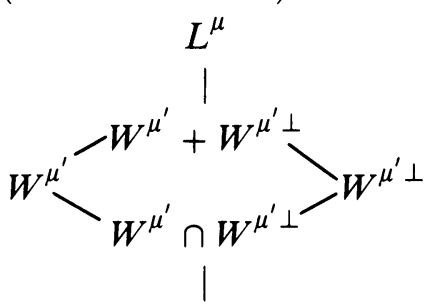

(0)

We see that the factors of $W^{\mu^{\prime}}$ are $F^{\mu^{\prime}}$ and some factors $F^{\lambda}$ with $\mu^{\prime} \triangleright \lambda$. Therefore, every composition factor of $L^{\mu}$ has the form $F^{\lambda}$ for some $\lambda$ with $\mu^{\prime} \unrhd \lambda$. (Consider the series $L^{\mu} \geq W^{\mu^{\prime}} \geq\left(W^{\mu^{\prime}} \cap W^{\mu^{\prime} \perp}\right)$ and recall that the factors of $L^{\mu} / W^{\mu^{\prime}}$ are the same as those of $W^{\mu^{\prime} \perp}$.) This completes the proof of $(*)$, and hence also the proof of the theorem.

8.9. Corollary. Suppose that $R$ is a field, and $\mu \in \Lambda(n, r)$. Then

(i) Every composition factor of $L^{\mu}$ has the form $F^{\lambda}$ for some $\lambda \vdash r$ with $\mu^{\prime} \unrhd \lambda$. The factor $F^{\mu^{\prime}}$ occurs with multiplicity one.

(ii) Every composition factor of $W^{\mu} \leq L^{\mu^{\prime}}$ has the form $F^{\lambda}$ for some $\lambda \vdash r$ and $\mu \unrhd \lambda$. The factor $F^{\mu}$ occurs with multiplicity one.

Proof. The result follows from the theorem and Corollary 4.10.

Finally, we note that if $N \geq n$, then we may follow the technique of Green [5, §6.5] to obtain results for $S_{R}(n, r)$ from the corresponding results for $S_{R}(N, r)$. In particular, this enables us to deal with the case when $n<r$.

Briefly, the method is the following. Suppose that $N \geq n$. Define the idempotent $e=\sum \phi_{\lambda \lambda}^{1} \in S_{R}(N, r)$, the sum being over all compositions $\lambda=$ $\left(\lambda_{1}, \lambda_{2}, \ldots, \lambda_{N}\right)$ of $r$ for which $\lambda_{n+1}=\cdots=\lambda_{N}=0$. Then $e S_{R}(N, r) e=$ $S_{R}(n, r)$, and each $S_{R}(N, r)$-module $U$ gives us an $S_{R}(n, r)$-module $e U$. In particular, the irreducible $S_{R}(n, r)$-modules are given by $\left\{e F^{\lambda} \mid \lambda \vdash r\right.$ and $\lambda$ has at most $n$ nonzero parts\}. Further details can be found in [5]. 


\section{REFERENCES}

1. R.W. Carter and G. Lusztig, On the modular representations of general linear and symmetric groups, Math. Z. 136 (1974), 193-242.

2. R. Dipper and G. D. James, Representations of Hecke algebras of general linear groups, Proc. London Math. Soc. (3) 52 (1986), 20-52.

3. _ Blocks and idempotents of Hecke algebras of general linear groups, Proc. London Math. Soc. (3) 54 (1987), 57-82.

4. —, The q-Schur algebra, Proc. London Math. Soc. (to appear).

5. J. A. Green, Polynomial representations of $G L_{n}$, Lecture Notes in Math., vol. 830, SpringerVerlag, 1980.

Department of Mathematics, The University of Oklahoma, Norman, OKLahoma 73019

IMPERIAL COLLEGE, LONDON SW7 2BZ, ENGLAND 